\title{
Atomic spin-wave control and spin-dependent kicks with shaped subnanosecond pulses
}

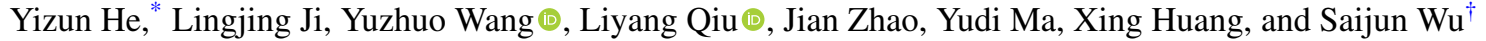 \\ Department of Physics, State Key Laboratory of Surface Physics and Key Laboratory of Micro \\ and Nano Photonic Structures (Ministry of Education), Fudan University, Shanghai 200433, China \\ Darrick E. Chang $\oplus^{*}$ \\ ICFO-Institut de Ciencies Fotoniques, The Barcelona Institute of Science and Technology, 08860 Castelldefels, Barcelona, Spain \\ and ICREA-Institució Catalana de Recerca i Estudis Avançats, 08015 Barcelona, Spain
}

(Received 26 April 2020; revised 1 December 2020; accepted 28 November 2020; published 24 December 2020)

\begin{abstract}
The absorption of traveling photons resonant with electric dipole transitions of an atomic gas naturally leads to electric dipole spin-wave excitations. For a number of applications, it would be highly desirable to shape and coherently control the spatial waveform of the spin waves before spontaneous emission can occur. This paper details a recently developed optical control technique to achieve this goal, where counterpropagating, shaped subnanosecond pulses impart subwavelength geometric phases to the spin waves by cyclically driving an auxiliary transition. In particular, we apply this technique to reversibly shift the wave vector of a spin wave on the $D 2$ line of laser-cooled ${ }^{87} \mathrm{Rb}$ atoms by driving an auxiliary $D 1$ transition with shape-optimized pulses, so as to shut off and recall superradiance on demand. We investigate a spin-dependent momentum transfer during the spin-wave control process, which leads to a transient optical force as large as $\sim 1 \hbar k / \mathrm{ns}$, and study the limitations to the achieved $70 \sim 75 \%$ spin-wave control efficiency by jointly characterizing the spin-wave control and matter-wave acceleration. Aided by numerical modeling, we project potential future improvements of the control fidelity to the $99 \%$ level when the atomic states are better prepared and by equipping a faster and more powerful pulse shaper. Our technique also enables a background-free measurement of the superradiant emission to unveil the precise scaling of the emission intensity and decay rate with optical depth.
\end{abstract}

DOI: 10.1103/PhysRevResearch.2.043418

\section{INTRODUCTION}

Spontaneous emission is typically a decoherence effect to avoid when levels in small quantum systems are chosen to encode information for, e.g., quantum computation, simulation, or sensing [1-4]. As spontaneous as it is, the information flow during the process can nevertheless be controlled between long-lived matter degrees of freedom and a prealigned single-mode electromagnetic continuum [5,6]. In particular, since the seminal work by Dicke in 1954 on super- and subradiant effects of light emission by ensembles of excited atoms [7], it is now well-known that the spatiotemporal properties of spontaneous emission are, in principle, dictated by collective properties of the atoms themselves. For spatially extended atomic ensembles, the timed phase correlations of the collective excitations, in the form of spin waves with phase-matched wave vector $\mathbf{k}$ satisfying $|\mathbf{k}|=$ $\omega / c$, can direct superradiant emission into narrow solid angles

\footnotetext{
*yzhe16@fudan.edu.cn

$\dagger^{\dagger}$ saijunwu@fudan.edu.cn

‡arrick.chang@icfo.eu
}

Published by the American Physical Society under the terms of the Creative Commons Attribution 4.0 International license. Further distribution of this work must maintain attribution to the author(s) and the published article's title, journal citation, and DOI.
[8-11]. This collective enhancement forms the basis for many applications predicated upon efficient quantum atom-light interfaces $[5,12]$. However, there has been relatively little work to address the question of what happens when $|\mathbf{k}| \neq \omega / c$ becomes strongly phase-mismatched from radiation. Within the context of atomic ensembles, complex phenomena can arise involving the combination of spatial disorder, multiple scattering of light, and dipole-dipole interactions between atoms [13-17], with much still left to be understood. Furthermore, within the emerging field of quantum optics with atomic arrays, such phase-mismatched states are predicted to be strongly subradiant. This forms the basis for exciting applications like waveguiding of light by the array [18-20], atomic mirrors [21-23], and exotic states [24,25], including emergent Weyl excitations [26] and topological guided edge modes [27,28], and the generation of highly correlated "fermionized" states [29]. One major bottleneck to exploring and controlling all these phenomena is the fact that any optical pulses used to manipulate atomic excited states, being radiation waves, most naturally excite phase-matched spin waves, while spin waves with $|\mathbf{k}| \neq \omega / c$ are naturally decoupled from light. What is needed then is a technique to efficiently and coherently alter the phase-matching condition of collective atomic excitations in the temporal domain - that is, to modify the wave vector of the spin waves and coherently convert between superradiant and subradiant modes-on rapid timescales faster than the typical emission time of atoms themselves. 
Previously, in a short Letter [30], we described an experimental realization of coherent dipole spin-wave control, based upon rapidly and cyclically driving an auxiliary transition with counterpropagating control pulses to robustly imprint spinand spatially dependent geometric phases onto the atoms. The resulting $\mathbf{k}$-space shifts lead to spin waves in a ${ }^{87} \mathrm{Rb}$ gas with strongly mismatched wave vectors $|\mathbf{k}| \neq \omega / c$ off the light cone and suppressed superradiant emission. Later, the mismatched spin waves are shifted back onto the light cone to cooperatively emit again on demand. In this paper, we expand upon key details that were previously omitted, describing in greater detail the experimental implementation and theoretical modeling of the geometric control technique, and additional research advances enabled by the technique. Furthermore, we carefully characterize the fidelity of our control sequence and discuss how current limitations can be overcome.

Beyond a detailed discussion of our spin-wave control technique, here we also give an example of its use toward the precise study of fundamental quantum optical phenomena. In particular, we exploit the ability to shift the dipole spin-wave vector to measure phase-matched forward collective emission in a background-free manner. The forward collective emission has been studied previously as a strong signature of cooperative enhancement of light-atom interactions in cold atomic gases $[10,11,15]$. One challenge to measure the forward cooperative emission in these experiments is related to the fact that the exciting beam, typically with a much stronger intensity, is in the same direction as the forward emission and contributes a large background. Here, the ability to shift the spin-wave vector in our case allows us to detect the forward superradiant emission from a different, background-free direction. We experimentally quantify the scaling of the emission intensity $i_{N} \propto N^{2}$ and collective decay rate $\Gamma_{N} / \Gamma=(1+\overline{\mathrm{OD}} / 4)$ $[13,16,17,25]$, for superradiant emission involving $N$ atoms with an average optical depth $\overline{\mathrm{OD}}$.

This paper also brings together two seemingly unrelated phenomena: the control of collective dipole radiation, and the acceleration of the free emitters. Accompanying the nanosecond spin-wave $\mathbf{k}$-vector shifts, we observe a strong spin-dependent optical force that accelerates the atomic sample at a $\sim 10^{7} \mathrm{~m} / \mathrm{s}^{2}$ transient rate. Similar techniques of cyclic rapid adiabatic passage have been studied in pioneering work by Metcalf et al. [31,32] as a robust way to impart strong optical forces to neutral atoms and molecules, with important applications in laser cooling $[33,34]$. The $\sim 1 \hbar k$ per nanosecond optical force in this work is among the highest $[33,35]$. The negative impacts by the spin-dependent acceleration to the spin-wave control is negligible in this work, and can be mitigated with lattice confinements in future experiments [30]. On the other hand, the combined effects may open interesting opportunities at the interface of quantum optics and atom interferometry [36].

The remainder of the paper is structured as follows. First, in Sec. II, we provide a simple, idealized theoretical description of our protocol to manipulate spin waves by rapid geometric phase patterning using shaped subnanosecond pulses. In Sec. III, we detail the experimental implementation of the coherent control and discuss the background-free detection of the cooperative emission as well as matter-wave acceleration effects. Here we also generalize the simpler theoretical discus- sion for quantifying the efficiency of our protocol in the face of various imperfections. We summarize this work in Sec. IV, and discuss methods and technology for improvements of the spin-wave control efficiency to the $\sim 99 \%$ level. To ensure completeness and to provide better context, key ideas from Ref. [30] are repeated in this paper.

\section{PRINCIPLES}

\section{A. Preparation and control of optical spin waves}

Single collective excitations of an atomic ensemble, consisting of $N$ atoms with ground state $|g\rangle$ and excited state $|e\rangle$, are naturally described by spin-wave excitations or timedDicke states of the form $\left|\psi_{\mathrm{TD}}\left(\mathbf{k}_{p}\right)\right\rangle=S^{+}\left(\mathbf{k}_{p}\right)\left|g_{1}, g_{2}, \ldots, g_{N}\right\rangle$. Here $S^{+}\left(\mathbf{k}_{p}\right)=\frac{1}{\sqrt{N}} \sum_{i} e^{i \mathbf{k}_{p} \cdot \mathbf{r}_{i}}\left|e_{i}\right\rangle\left\langle g_{i}\right|$ denotes a collective spin raising operator and $\mathbf{k}_{p}$ the corresponding wave vector. For example, a weak coherent state involving such excitations is naturally generated by an incoming plane-wave probe pulse with Rabi frequency $\Omega_{p}$ and duration $\tau_{p} \ll 1 / \Omega_{p}$, if $\tau_{p}$ is in addition short enough that light rescattering effects are negligible. As such, the magnitude of the wave vector $\left|\mathbf{k}_{p}\right|=\omega_{e g} / c$, with $\omega_{e g}$ being the atomic resonance frequency, matches that of free-space radiation. As is well-known [9-11], such phasematched spin-wave excitations in a large sample with size $\sigma \gg 1 / k_{p}$ and $N>k_{p}^{2} \sigma^{2}$ radiate efficiently and in a collectively enhanced fashion, much like a phased array antenna, into a small solid angle $\theta^{2}$ with $\theta \sim 1 / k_{p} \sigma$ around the forward $\mathbf{k}_{p}$ direction.

We are interested in spin waves with strongly mismatched $|\mathbf{k}| \neq \omega_{e g} / c$ off the light cone, in which case the cooperative emission is prohibited. In an ensemble with random atomic positions $\left\{\mathbf{r}_{i}\right\}$, as in this paper, such a spin wave is expected to decay with a rate near the natural linewidth [37], as the fields emitted by different atoms in any direction average to zero, but with nonzero variance. On the other hand, in an ordered array of atoms, the destructive interference in all directions can be nearly perfect, leading to strong subradiance that forms the basis of the exciting applications mentioned earlier [18-28]. It is therefore highly compelling to have a technique to shift the spin waves in $\mathbf{k}$ space on a timescale much faster than the spontaneous emission lifetime. To achieve the required $\mathbf{k}$-vector shift, we consider a unitary transform $U_{c}(\Delta \mathbf{k})$ such that $S^{+}(\mathbf{k}+\Delta \mathbf{k})=U_{c}(\Delta \mathbf{k}) S^{+}(\mathbf{k}) U_{c}^{\dagger}(\Delta \mathbf{k})$. The required control is a class of state-dependent phase-patterning operations, which can be decomposed into spatially dependent phase gates for each two-level atom as (with $\sigma_{z, i}=\left|e_{i}\right\rangle\left\langle e_{i}\right|-$ $\left.\left|g_{i}\right\rangle\left\langle g_{i}\right|\right)$

$$
U_{c}(\Delta \mathbf{k})=\prod_{i}^{N} e^{\frac{i}{2} \Delta \mathbf{k} \cdot \mathbf{r}_{i} \sigma_{z, i}} .
$$

While we have thus far focused on the manipulation of spin-wave excitations by considering the position $\mathbf{r}_{i}$ as an operator as well, one sees that the transformation of Eq. (1) also imparts opposite momentum boosts to the $g, e$ components of a freely moving atom. Techniques for realizing such spindependent kicks have been well-developed in the community of atom interferometry and ion-based quantum information processing typically on Raman transitions [38-40]. Here, we demonstrate a high-efficiency process based upon rapid 

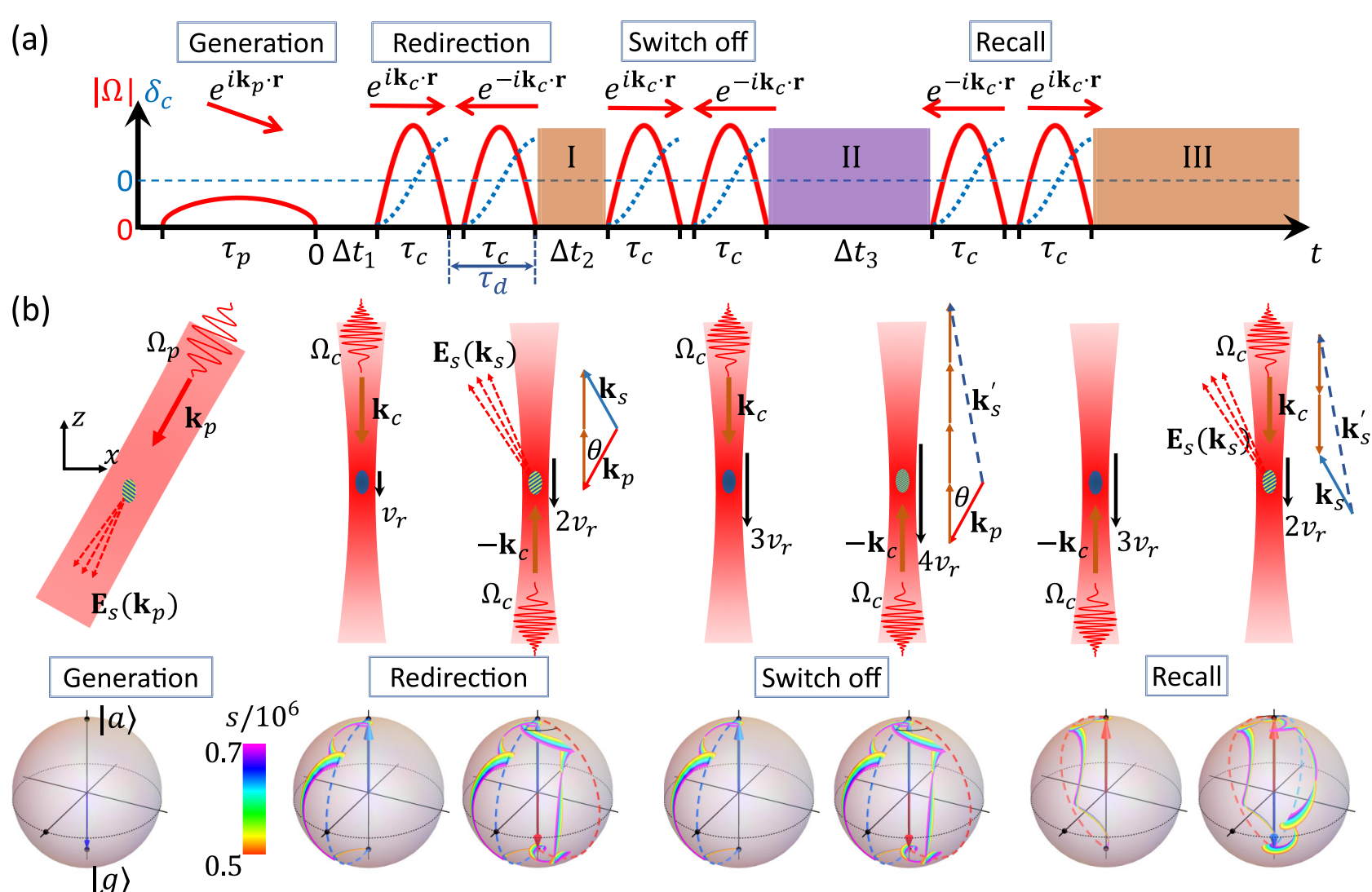

FIG. 1. Schematic of the experiment to demonstrate error-resilient optical spin-wave control. (a) Schematic timing sequence for the amplitudes of the probe and control pulse Rabi frequency $|\Omega|$ (red solid lines), and the instantaneous detuning of the control pulse $\delta_{c}$ (blue dashed lines) from the $|g\rangle-|a\rangle$ transition. (b) Top (from left to right): Generation and control of optical spin wave with the probe beam followed by the control pulse-induced redirection, switch off and recall of the collective spontaneous emission. The $|g\rangle-|e\rangle$ electric dipole spin wave is illustrated with fringes in the atomic sample. Each optical control also imparts a spin-dependent kick, which leads to momentum transfer with $v_{r}=\hbar k_{c} / m \approx 5.8 \mathrm{~mm} / \mathrm{s}, m$ being the atomic mass of ${ }^{87} \mathrm{Rb}$. The drawings are not to actual scale, in particular, the phase-matching angle $\theta=\arccos \frac{\lambda_{D 2}}{\lambda_{D 1}} \sim 11.1^{\circ}$ is exaggerated for clarity. Bottom: Bloch-sphere representation of the projected $|g\rangle-|a\rangle$ state dynamics for an atom at a representative position $\mathbf{r}$. An ensemble of trajectories with different control pulse peak intensity parameters $s$ is displayed. The quasiadiabatic control ensures that the geometric phase writing is insensitive to small deviations of $s$ from $s \sim 0.6 \times 10^{6}$, for $\tau_{c} \Gamma_{D 1}=0.02 \sim 0.03$ in this paper.

manipulation of a strong optical transition for the spin-wave control.

\section{B. Error-resilient spin-wave control}

To implement $U_{c}(\Delta \mathbf{k})$ in Eq. (1) on a strong dipole transition in a large atomic sample, we combine the geometric phase method suggested in Ref. [37] with optical rapid adiabatic passage techniques as in Refs. [31,32] for the necessary control speed, precision, and intensity-error resilience. In particular, we consider two nearly identical optical "control" pulses with Rabi frequency $\Omega_{c}(t) e^{i \varphi_{1,2}}$ and instantaneous detuning $\delta_{c}(t)$ to drive the auxiliary $|g\rangle \rightarrow|a\rangle$ and $|a\rangle \rightarrow|g\rangle$ transitions, respectively. By optical pulse shaping, the cyclic transition can be driven with high precision in a manner that is largely insensitive to laser intensity. We adapt a simple choice of $\Omega_{c}(t)=\Omega_{0} \sin \left(\pi t / \tau_{c}\right)$ and $\delta_{c}(t)=-\delta_{0} \cos \left(\pi t / \tau_{c}\right)$ to achieve quasiadiabatic population inversions at optimal $\left\{\Omega_{0}, \delta_{0}\right\}$ within the $\tau_{c}$ pulse duration $[31,32]$. To connect to the experimental setup (Figs. 1 and 2), after preparing the $S^{+}\left(\mathbf{k}_{p}\right)$ spin-wave excitation, we send the first control pulse to the atomic sample, propagating along the direction given by wave vector $\pm \mathbf{k}_{c}$, driving $|g\rangle \rightarrow|a\rangle$. Subsequently, a second control pulse with $\mp \mathbf{k}_{c}$ interacts with the sample after a $\tau_{d}$ delay, driving $|a\rangle \rightarrow|g\rangle$. Although every ground-state atom in the original $|g\rangle-|e\rangle$ spin wave returns to $|g\rangle$, the area enclosed around the Bloch sphere by the state vector causes each atom to pick up a spatially dependent geometric phase $\varphi_{\mathrm{G}}(\mathbf{r})=\pi+\Delta \mathbf{k} \cdot \mathbf{r}_{i}$, with $\Delta \mathbf{k}= \pm 2 \mathbf{k}_{c}$ to fully exploit the resolution of the optical phase. The ideal state-dependent phase patterning achievable in the short $\tau_{c, d}$ limit can be formally expressed within the $\{|g\rangle,|e\rangle\}$ space as

$$
U_{g}(\Delta \mathbf{k})=\prod_{i}^{N}\left(\left|e_{i}\right\rangle\left\langle e_{i}\left|-e^{i \Delta \mathbf{k} \cdot \mathbf{r}_{i}}\right| g_{i}\right\rangle\left\langle g_{i}\right|\right),
$$

which performs a $\mathbf{k} \rightarrow \mathbf{k}-\Delta \mathbf{k}$ shift to spin-wave excitations the same way as $U_{c}(-\Delta \mathbf{k})$ in Eq. (1).

Although the phase patterning operation in Eq. (2) could in principle be achieved without the control pulse shaping, practically, the control laser intensity inhomogeneity across the large sample would translate directly into spin control 
(a)

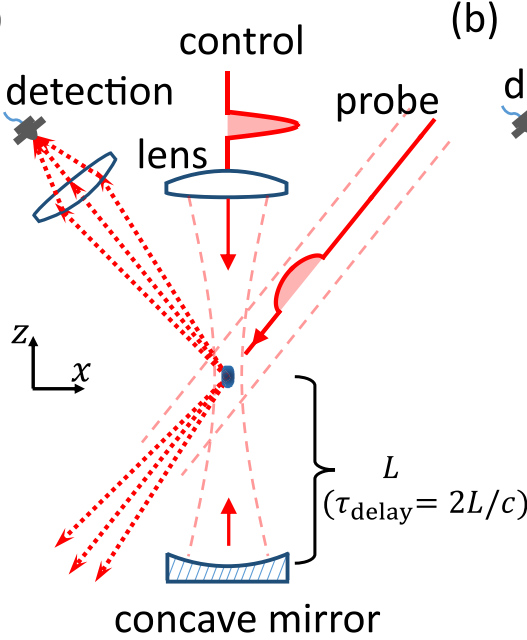

(b)

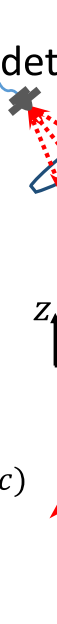

(c)

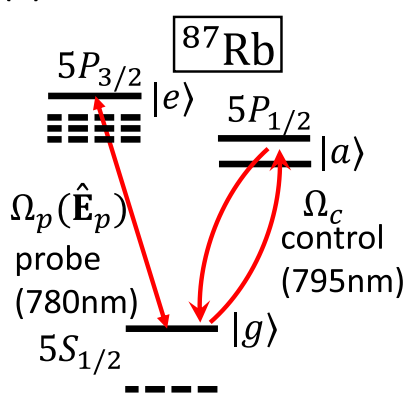

FIG. 2. Setup schematics for spin-wave control based on optically delayed retroreflections. The schematics share the same relative orientations of the laser beams as in Fig. 1 . In (a), the incoming pulse is retroreflected with a delay $\tau_{\text {delay }}=1.36 \mathrm{~ns}$ for $L \approx 200 \mathrm{~mm}$ to interact twice with the atomic sample. In (b), the preprogrammed pulse (marked with $p^{\prime}$ ) is stored in the much longer optical delay line with $L \approx 15 \mathrm{~m}$ and $\tau_{\text {delay }} \approx 100 \mathrm{~ns}$, timed to collide with a new incoming pulse (marked with $p$ ). (c) The $D 1$ and $D 2$ level diagram for the ${ }^{87} \mathrm{Rb}$ with hyperfine structures and the laser coupling scheme in this paper.

error, as in most nonlinear spectroscopy experiments [41-43], leading to degraded average control fidelity if a highly uniform laser intensity profile cannot be maintained. To fully exploit the intensity-error resilience [44] offered by the SU(2) geometry of the two-level control, the optical pulses need to be shaped on a timescale fast enough to suppress the spontaneous emission, and also slow enough to avoid uncontrolled multiphoton couplings in real atoms. We note that optical methods for two-level rapid adiabatic passage [45] itself are welldeveloped for population transfers in atoms and molecules using ultrafast lasers $[46,47]$. However, these ultrafast techniques typically demand control field Rabi frequencies $\Omega_{c}$ beyond the $\mathrm{THz}$ level with intense pulses at low repetition rates, not easily adaptable to our desired goals. The strong fields may also cause non-negligible multilevel couplings or even photoionization beyond the desired multiple population inversions. Instead of using ultrafast lasers, here we develop a wide-band pulse-shaping technique based on fiber-based sideband electro-optical modulation of a cw laser [30], with up to $13 \mathrm{GHz}$ modulation bandwidth, to support the flexibly programmable error-resilient spin wave control. Compared with previous work on spectroscopy based upon perturbative nonlinear optical effects [41-43], our technique is unique in that we steer the atomic state over the entire Bloch sphere of the two-level system to achieve the geometric robustness toward perfect spin-wave control set by Eq. (2).

\section{Dynamics of controlled emission}

Here we go beyond Ref. [30] to discuss the expected emission characteristics of phase-matched and mismatched spin waves, which will constitute one of the key observables to verify our coherent control technique and quantify its efficiency. Later, in Sec. III B, we will experimentally verify the predicted optical depth dependence for the phase- matched case. To specifically relate to experimental control of a laser-cooled gas in this work, we consider an atomic sample with a smooth profile $\varrho(\mathbf{r})=\left\langle\sum_{i} \delta\left(\mathbf{r}-\mathbf{r}_{i}\right)\right\rangle$ nearly spherical with size $\sigma \gg 1 /\left|\mathbf{k}_{p}\right|$ and at a moderate density with $\varrho<\left|\mathbf{k}_{p}\right|^{3}$. Formally, the quantum field emitted by a collection of atoms on the $|e\rangle-|g\rangle$ transition can be expressed in terms of the atomic spin coherences themselves, $\hat{\mathbf{E}}_{s}(\mathbf{r})=$ $\omega_{e g}^{2} / \varepsilon_{0} c^{2} \sum_{i} \mathbf{G}\left(\mathbf{r}-\mathbf{r}_{i}, \omega_{e g}\right) \cdot \mathbf{d}_{e g}\left|g_{i}\right\rangle\left\langle e_{i}\right|$ [24]. Here $\mathbf{G}\left(\mathbf{r}, \omega_{e g}\right)$ is the free-space Green's tensor of the electric field, which physically describes the field at position $\mathbf{r}$ produced by an oscillating dipole at the origin, and $\mathbf{d}_{e g}=d_{e g} \mathbf{e}_{d}$ is the transition dipole moment. For the single-excitation timed Dicke state, one can define a single-photon wave function $\boldsymbol{\varepsilon}_{\mathbf{k}}(\mathbf{r})=$ $\left\langle g_{1}, g_{2}, \ldots, g_{N}\left|\hat{\mathbf{E}}_{s}\right| \psi_{\mathrm{TD}}(\mathbf{k})\right\rangle$, which describes the spatial profile of the emitted photon. Of particular interest will be the field emitted along direction $\mathbf{k}$ at the end of the nearly spherical sample, and at a transverse position $\mathbf{r}_{\perp}$. Within the so-called Raman-Nath regime where diffraction is negligible, we obtain $\overline{\varepsilon_{\mathbf{k}}}(\mathbf{r})$ averaged over random $\left\{\mathbf{r}_{i}\right\}$ as (also see Appendix B)

$$
\overline{\varepsilon_{\mathbf{k}}}(\mathbf{r}) \approx \mathbf{e}_{d} \sqrt{\frac{\hbar \omega_{e g}}{8 \varepsilon_{0} c}} \sqrt{N \sigma_{r} \Gamma} \varrho_{c}\left(\mathbf{r}_{\perp}, \delta k\right) e^{i \omega_{e g} r_{\mathbf{k}} / c} .
$$

Here $r_{\mathbf{k}}=\mathbf{r} \cdot \mathbf{k} /|\mathbf{k}|$, and $\varrho_{c}\left(\mathbf{r}_{\perp}, \delta k\right)=\frac{1}{N} \int \varrho(\mathbf{r}) e^{i \delta k r_{\mathbf{k}}} d r_{\mathbf{k}}$ is a generalized (and normalized) column density, where in general we allow for a wave number $\delta k=|\mathbf{k}|-\omega_{e g} / c$ that is mismatched from radiation. $\sigma_{r}=\omega_{e g} \alpha_{i} / c$ is the resonant absorption cross section, and the imaginary part of the resonant polarizability $\alpha_{i}$ is related to the dipole element $\mathbf{d}_{e g}$ and $\Gamma$ through $\hbar \Gamma \alpha_{i}=2\left|\mathbf{d}_{e g}\right|^{2}$. While $\mathbf{d}_{e g}$ and $\Gamma$ are directly related for two-level atoms, this formula also generalizes to atoms with level-degeneracy.

Equation (3) describes the possibility of both enhanced or suppressed collective emission associated with the spinwave excitation at a $\delta k$ radiation wave-number mismatch. A 
well-known consequence of Eq. (3) is that when the spin-wave excitation is by a weak probe pulse with wave vector $\mathbf{k}_{p}$ (see Fig. 1), the atoms act as a phased antenna array with $\delta k=0$, and light in the forward direction along $\mathbf{k}_{p}$ is re-emitted at an enhanced rate within an angle $\theta \sim 1 /\left(\left|\mathbf{k}_{p}\right| \sigma\right)$ [48]. As our probe pulse is in a weak coherent state, the timed Dicke state is excited with a population of $N \theta_{p}^{2}$, where $\theta_{p}=\frac{1}{2} \int \Omega_{p} d t$ is the time-integrated Rabi frequency of the probe pulse $\left(\theta_{p} \ll 1\right)$. Assuming that the spatial profile of Eq. (3) does not change significantly during the emission process, one can integrate the intensity of light predicted by Eq. (3) over space, and arrive at the following time-dependent collective spontaneous emission rate:

$$
i_{\mathbf{k}_{p}}(t) \approx N \theta_{p}^{2}\left(\overline{\mathrm{OD}}_{p} / 4\right) \Gamma e^{-\left(1+\overline{\mathrm{OD}}_{p} / 4\right) \Gamma t} .
$$

Here $\overline{\mathrm{OD}}_{p} \equiv \int \mathrm{OD}_{p}^{2}\left(\mathbf{r}_{\perp}\right) / \int \mathrm{OD}_{p}\left(\mathbf{r}_{\perp}\right) \propto N$ is the average optical depth, and $\mathrm{OD}_{p}\left(\mathbf{r}_{\perp}\right)=N \varrho_{c}\left(\mathbf{r}_{\perp}, \delta k=0\right) \sigma_{r}$. The exponential factors of $e^{-\Gamma t}$ and $e^{-\overline{\mathrm{OD}}_{p} \Gamma t / 4}$ account for the (noncollective) decay into $4 \pi$ and enhanced emission along the phase-matched $\mathbf{k}_{p}$ direction, respectively.

We now consider what happens if, immediately following the probe pulse at $t=0$, we apply the ideal spin-wave control, which imprints a geometric phase and transforms the original timed-Dicke state along $\mathbf{k}_{p}$ according to Eq. (2) (finite delay times and other imperfections can be straightforwardly included, as discussed in Sec. III). As the original state has a well-defined wave vector, the application of Eq. (2) simply creates a new timed Dicke state with new wave vector $\mathbf{k}_{s}=\mathbf{k}_{p}-2 \mathbf{k}_{c}$. Then, two distinct cases emerge. The first is that $\left|\mathbf{k}_{s}\right| \approx \omega_{e g} / c$, in which case the spin wave is again phase matched to radiation and an enhanced emission rate like Eq. (4) is again observed, but with the majority of emission "redirected" along the new direction $\mathbf{k}_{s}$. The second, and more intriguing, possibility is that $\left|\mathbf{k}_{s}\right|$ is significantly mismatched from $\omega_{e g} / c$. In that case, there is no direction along which emission can be constructively and collectively enhanced. For the case of our disordered ensemble, this results in the emission rate into the same solid angle in absence of phase-matching as

$$
i_{\mathbf{k}_{p}}^{\prime}(t) \approx N \theta_{p}^{2} /\left(\left|\mathbf{k}_{p}\right|^{2} \sigma^{2}\right) \Gamma e^{-\Gamma t},
$$

i.e., the emission reduces to an incoherent sum of those from single, isolated atoms. This is due to the random positions of the atoms, such that the field of emitted light in any direction tends to average to zero, but with a nonzero variance [corrections to Eq. (5) are expected at high densities due to granularity of the atomic distribution, which make the problem quite complex in general $[13,14,49]]$. However, in an ordered array of atoms, the destructive interference in all directions can be nearly perfect, leading to a decay rate much smaller than $\Gamma$. The ability to generate excited states with extremely long lifetimes is key to all of the applications mentioned in the Introduction [18-24,26-28,50].

\section{EXPERIMENTAL RESULTS}

\section{A. Experimental methods}

In this paper, the dipole spin-wave excitation is implemented on the ${ }^{87} \mathrm{Rb} 5 S_{1 / 2}-5 P_{3 / 2} \quad D 2$ line between hyperfine ground state $5 S_{1 / 2} F=2$ and excited state $5 P_{3 / 2} F^{\prime}=3$, represented by $|g\rangle$ and $|e\rangle$ in Fig. 2, respectively. The transition wavelength is $\lambda_{\mathrm{D} 2}=780 \mathrm{~nm}$ (with $\left.k_{p}=2 \pi / \lambda_{\mathrm{D} 2}\right)$, and the natural linewidth is $\Gamma_{\mathrm{D} 2}=2 \pi \times 6.07$ MHz. We prepare $N \sim 10^{487} \mathrm{Rb}$ atoms in $|g\rangle$ in an optical dipole trap with up to $\sim 5 \times 10^{12} / \mathrm{cm}^{3}$ peak density and $T \sim 20 \mu \mathrm{K}$ temperature. After the atoms are released from the trap, the dipole excitation is induced by a $\tau_{p}=3 \sim 5 \mathrm{~ns}$, $I_{p} \approx 10 \mathrm{~mW} / \mathrm{cm}^{2}$ resonant $D 2$ probe pulse. The Gaussian probe beam has a $w_{p} \approx 50 \mu \mathrm{m}$ waist, which is much larger than the $1 / e$ radius of atomic density profile $\sigma \approx 7 \mu \mathrm{m}$, validating the plane-wave excitation picture.

The auxiliary transition is implemented on the $D 1$ line between $|g\rangle$ and $|a\rangle$, with $|a\rangle$ representing the $5 P_{1 / 2} F^{\prime}=$ 1,2 levels, with $\lambda_{D 1}=795 \mathrm{~nm}, k_{c}=2 \pi / \lambda_{D 1}$ and $\Gamma_{D 1}=$ $2 \pi \times 5.75 \mathrm{MHz}$. The spin wave $U_{g}$ control as in Eq. (2) is implemented by cyclically driving the $D 1$ transition with the counter-propagating chirped pulses, with Rabi frequency $\Omega_{c}(t)=\Omega_{0} \sin \left(\pi t / \tau_{c}\right)$ and instantaneous detuning $\delta_{c}(t)=$ $-\delta_{0} \cos \left(\pi t / \tau_{c}\right)$ (defined relative to the midpoint of the $5 S_{1 / 2} F=2-5 P_{1 / 2} F^{\prime}=1,2$ hyperfine lines), so as to phase pattern the $5 S_{1 / 2} F=2$ atoms without perturbing the $5 P_{3 / 2}$ level due to the large $D 1-D 2$ transition frequency difference. Utilizing our subnanosecond pulse-shaping technology as detailed in Ref. [30], with $\sim 20 \mathrm{~mW}$ of peak power, peak intensity parameter $s \sim 10^{6}\left(s \equiv I / I_{s 1}\right.$ and $I_{s 1}=4.49 \mathrm{~mW} / \mathrm{cm}^{2}$ is the $D 1$ transition saturation intensity [51]) and peak Rabi frequency $\Omega_{0}=\sqrt{s / 2} \Gamma_{D 1}$ at $\mathrm{GHz}$ level are reached by focusing the $\mathbf{k}_{c}$-control beam into a waist of $w \approx 13 \mu \mathrm{m}$ at the atomic sample.

We choose the polarization for the probe and control lasers to be along $\mathbf{e}_{y}$ and $\mathbf{e}_{x}$, respectively. Taking the $\mathbf{e}_{x}$-direction as the quantization axis, the $\pi$-control couplings to $5 P_{1 / 2}$ would be with equal strengths and detunings for all five $5 S_{1 / 2} F=$ $2, m_{F}$ Zeeman sublevels, and with vanishing hyperfine Raman coupling, if the $5 P_{1 / 2}$ hyperfine splitting $\Delta_{D 1 \text {, hfse }}=2 \pi \times$ 814.5 $\mathrm{MHz}$ can be ignored. The approximation helps us to establish the simple two-level control picture in Fig. 2(c), even for the real atom. Practically, the hyperfine dephasing effects can be suppressed for atoms in the $\left|m_{F}\right|=0$, 2 Zeeman sublevels by adjusting the optical delay $\tau_{d}$ to match $2 \pi / \Delta_{D 1 \text {,hfse }} \approx 1.23 \mathrm{~ns}$. The hyperfine effect more severely impacts the $\left|m_{F}\right|=1$ atoms through both intensity-dependent dephasing and nonadiabatic population losses. These hyperfine effects are suppressible with better Zeeman-state preparations or by faster controls with $\tau_{c} \Delta_{D 1 \text {,hfse }} \ll 1$ while setting $\tau_{d}$ to be a multiple of $2 \pi / \Delta_{D 1 \text {,hfse }}$.

To experimentally implement the spin-wave $U_{g}(\Delta \mathbf{k})$ control as in Eq. (1) with $\Delta \mathbf{k}= \pm 2 \mathbf{k}_{c}$, counterpropagating pulses are sent to the atomic sample using retroreflection with optical delay lines [Figs. 2(a) and 2(b)]. The ability to control the sign is important for the turnoff and recall of superradiance. Going beyond Ref. [30], we discuss how to implement this using two types of optical delays. In the first type [Fig. 2(a)], an incoming pulse and its retroreflection by a $R=200 \mathrm{~mm}$ concave mirror at a $L \approx 200 \mathrm{~mm}$ distance outside the vacuum interact twice with the sample with a $\tau_{\text {delay }}=2 L / c=$ $1.36 \mathrm{~ns}$ relative delay. This design conveniently enables the $|g\rangle \rightarrow|a\rangle \rightarrow|g\rangle$ cyclic transition driven by a 
$\mathbf{k}_{c}$ and then a $-\mathbf{k}_{c}$ pulse, thereby accomplishing a $U_{g}\left(2 \mathbf{k}_{c}\right)$ operation with nearly identical pulse pairs. However, the ordered arrivals for the $\pm \mathbf{k}_{c}$ pulses rule out the possibility of realizing efficient $U_{g}\left(-2 \mathbf{k}_{c}\right)$ control.

To reverse the time order for the $\pm \mathbf{k}_{c}$ pulses, we introduce a second type of optical delay as in Fig. 2(b). Here the delay distance $L \approx 15 \mathrm{~m}$ and the associated delay time $\tau_{\text {delay }} \approx$ $100 \mathrm{~ns}$ are long enough that we are able to temporally store a few preprogrammed pulses on the side of the atomic sample opposite to the incoming direction. In particular, before the preparation of the $|g\rangle-|e\rangle$ spin waves, up to three preprogrammed $D 1$ control pulses, such as the one marked with " $p$ " " in Fig. 2(b), are initially sent to the optical delay line. After all these pulses pass through the atomic sample, the atoms are excited and then decay into the $F=1,2$ ground states within the delay time $\tau_{\text {delay }} \gg 1 / \Gamma_{D 1}$. At the moment when these stored pulses are coming back, additional control pulses [such as the one marked with $p$ in Fig. 2(b)] with readjusted pulse properties are sent to collide with the preprogrammed pulses to form the control sequence including spin-wave controls $U_{g}\left( \pm 2 \mathbf{k}_{c}\right)$ in a designed order [Fig. 1(a)]. The second optical delay method is therefore more flexible for parametrizing the control pulse pairs to reversibly shift the spin waves in $\mathbf{k}$-space on demand. We notice that the first pass of the stored optical pulses causes some atom losses (to the $F=1$ ground states which are dark to the following spin-wave excitations). The amount of loss is a function of the (unwanted) $D 1$ excitation efficiency, and is therefore correlated to the pulse number, relative delays and shapes. By combining proper timing of the stored pulses with numerical modelings, the loss effects can be controlled in measurements where the atom numbers are important [49]. In addition, in this work the extra beam steering optics to fold the delay line onto the optical table introduces extra optical power loss (30\%) to the retroreflected pulses, challenging the range of intensity resilience for the control operation (also see Figs. 1 and 6). Therefore, to optimally operate the control with the second mode of optical delay, we typically readjust the pulse-shaping parameters and in particular to balance the power of the pulse pairs. To precisely measure the optical delay time $\tau_{\text {delay }}$, a single $D 1$ control chirped pulse is sent to the optical path to efficiently excite the atomic sample twice. The two fluorescence signals separated by $\tau_{\text {delay }}$ are collected to precisely measure $\tau_{\text {delay }}$ with $\sim 0.1 \mathrm{~ns}$ accuracy.

Additional details on the experimental measurements are given in Appendix A. With the experimental methods, we can precisely perform multiple $D 1$ controls to shift the dipole spin-wave excitation $S^{+}(\mathbf{k})$ on the $D 2$ transition from the original value of $\mathbf{k}=\mathbf{k}_{p}$ to the new wave vectors $\mathbf{k}_{p}$ $2 n \mathbf{k}_{c}$ (with $n=1,2$ in this paper) in a reversible manner. To benchmark the control quality, we set up the photon detection path along a finely aligned $\mathbf{k}_{s}=\mathbf{k}_{p}-2 \mathbf{k}_{c}$ direction to meet the $\left|\mathbf{k}_{s}\right|=\omega_{e g} / c$ redirected phase-matching condition (Fig. 1). As such, following the $\mathbf{k}_{p}$ spin wave preparations a $\mathbf{k} \rightarrow \mathbf{k}-2 \mathbf{k}_{c}$ shift can redirect the forward superradiance to $\mathbf{k}_{s}$ for its background-free detection. We collect the $\mathbf{k}_{s}$-mode superradiance with a NA=0.04 objective for detection by a multimode fiber coupled single photon counter. To enhance the measurement accuracy, an optical filter at
$780 \mathrm{~nm}$ is inserted to block possible fluorescence photons at $\lambda_{D 1}=795 \mathrm{~nm}$.

\section{B. Intensity and decay of the redirected emission}

The k-vector shift of dipole spin waves allows us to access both the subradiant states with strongly phase-mismatched wave vectors $\left|\mathbf{k}_{s}^{\prime}\right| \neq \omega_{e g} / c$, and the redirected superradiant states with phase-matched wave vectors $\left|\mathbf{k}_{s}\right|=\omega_{e g} / c$. A study on the dynamics of subradiant states is left for a future publication [49]. In this paper, we primarily focus on the dynamics of the directional superradiance. In particular, the redirected superradiant emission from $\mathbf{k}_{p}$ to $\mathbf{k}_{s}$ in our setup naturally avoids the large probe excitation background, which commonly exists in previous studies of forward emission [11,15], and thereby enables us to characterize the redirected superradiance with high accuracy. With the spin-wave excitation of timed-Dicke states prepared in a nearly ideal way (Sec. II A), we go beyond Ref. [30] and verify the $i_{\mathbf{k}_{p}} \propto N^{2}$ scaling as in Eq. (4). Furthermore, we observe possible deviation of the collective decay rate from $(1+\overline{\mathrm{OD}} / 4) \Gamma[13,16,17,25]$, which is likely related to a subtle superradiance reshaping effect [52].

We note Eq. (4) can describe what would be observed in the redirected emission in an ideal experiment, e.g., if the first pair of control pulses could be applied immediately $\left(\Delta t_{1}=0\right)$ and perfectly after the probe pulse. To quantitatively describe the actual experiment, however, we must account for the fact that the spin wave already begins decaying in a superradiant fashion along the direction $\mathbf{k}_{p}$ during the delay time $\Delta t_{1}$, that the ensemble is nonspherical and has different optical depths $\mathrm{OD}_{p}, \mathrm{OD}_{s}$ along the directions $\mathbf{k}_{p, s}$, and that the control efficiency $f_{d}<1$ for the applied unitary operation of Eq. (2) is not perfect. The nonideal $U_{g}$ control in presence of, e.g., $m_{F}$-dependent hyperfine phase shifts and spontaneous emission during the control, only partly converts the $S^{+}\left(\mathbf{k}_{p}\right)$ into $S^{+}\left(\mathbf{k}_{s}\right)$ excitation and further induces subwavelength density modulation in $\varrho(\mathbf{r})$, thus we expect simultaneous and Bragg-scattering coupled superradiant emission into both the $\mathbf{E}_{s}\left(\mathbf{k}_{p}\right)$ and $\mathbf{E}_{s}\left(\mathbf{k}_{s}\right)$ modes. Practically for optical control with the focused laser beam as in this work, we numerically find the ground-state atoms not shifted in momentum space are often associated with dynamical phase broadening, leading to suppressed $S^{+}\left(\mathbf{k}_{p}\right)$ excitation and distorted subwavelength density fringes. A simple model for the superradiant photon emission rate into the redirected $\mathbf{E}_{s}\left(\mathbf{k}_{s}\right)$ mode is

$$
i_{s}(t) \approx f_{d} \frac{\overline{\mathrm{OD}}_{s}}{\overline{\mathrm{OD}}_{p}} i_{p}\left(\Delta t_{1}\right) e^{-\left(1+(1-l) \overline{\mathrm{OD}}_{s} / 4\right) \Gamma_{D 2}\left(t-\Delta t_{1}\right)} .
$$

Here, we have accounted for the different optical depths along the $\mathbf{k}_{s, p}$ directions, the finite control efficiency $f_{d}$, the superradiant decay that already occurs during the time $\Delta t_{1}$ along the original direction $\mathbf{k}_{p}$, and the fraction of atoms $l$ that are lost during the control process.

Experimentally, the spin-wave control for superradiance redirection with a $\mathbf{k}$ vector shift $\mathbf{k}_{p} \rightarrow \mathbf{k}_{p}-2 \mathbf{k}_{c}$ by a single pair of control pulses is first demonstrated in Fig. 3(a), where the $\mathbf{E}_{s}\left(\mathbf{k}_{s}\right)$ emission at a fixed delay $\Delta t_{1}=0.2 \mathrm{~ns}$ is recorded. To study the collective effect of forward emission, we vary the atom number $N$ for atomic samples loaded into the same dipole trap with nearly identical spatial distribution. 
(a)

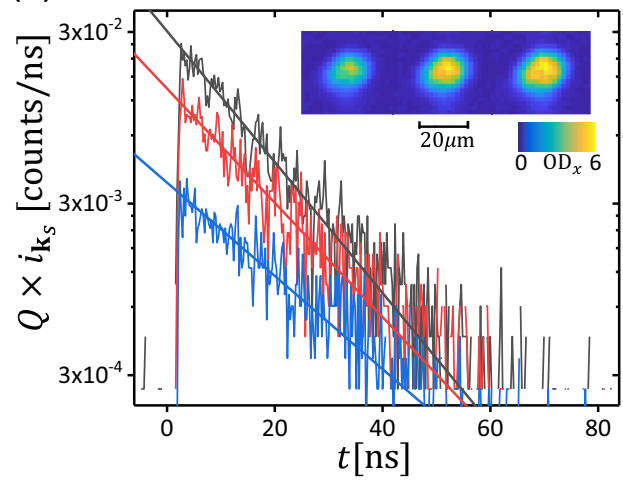

(b)

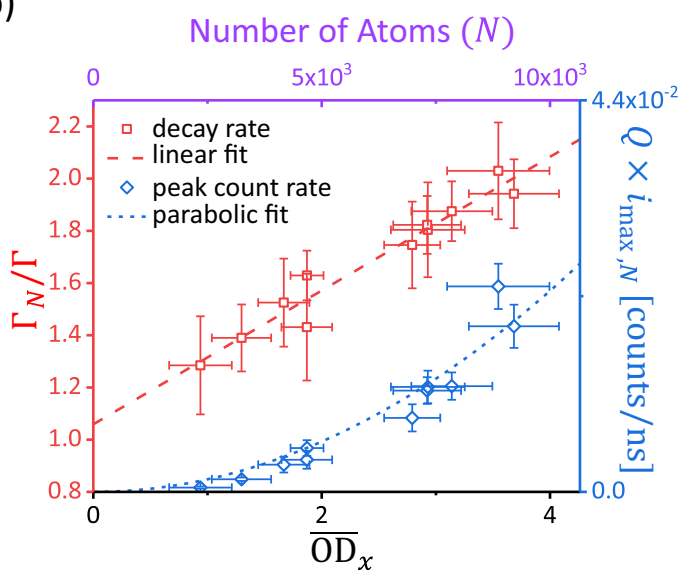

FIG. 3. (a) Typical time-dependent redirected superradiant emission rate $i_{s}(t)$ multiplied by a detection quantum efficiency $Q \approx 0.15$, estimated over $N_{\text {exp }}=13000$ experiments. The plot is on log scale. The curves are for samples with different atom numbers, with resonant optical depth images along $\mathbf{e}_{x}$ (insets) inferred from absorption images taken at the corresponding experimental conditions. The exponential fit gives the peak count rate $i_{\max , N}$ and the collective decay rate $\Gamma_{N}$. (b) $i_{\max , N}$ and $\Gamma_{N}$ are plotted versus estimated atom number $N$ and average optical depth $\overline{\mathrm{OD}}_{x}$. The error bars represent the statistical and fit uncertainties of the data.

The time-dependent photon emission rate $i_{s}(t)$, obtained by normalizing the fluorescence counts with the number of runs $N_{\text {exp }}$, counter time bin $\delta t$, and an overall detection quantum efficiency $Q \approx 0.15$, nicely follows exponential decay curves for the accessed $N$ between $2 \times 10^{3}$ and $9 \times 10^{3}$ in this work. We extract both the peak emission rate $i_{\max , N}$ and collective decay rate $\Gamma_{N}$ with exponential fits, and study both quantities as a function of atom number $N$.

The cooperative nature of the collective emission is clearly demonstrated in Fig. 3(b) with the $i_{\max , N} \propto N^{2}$ scaling since according to Eqs. (4) and (6) we have $i_{\max , N} \propto N \overline{\mathrm{OD}}_{s}$ but $\overline{\mathrm{OD}}_{s} \approx N \sigma_{r} / \sigma^{2}$ for our nearly spherical sample with size $\sigma$. We experimentally extrapolate the average optical depth $\overline{\mathrm{OD}}_{s}$ with $\mathrm{OD}_{x}(y, z)$ measurements along the $x$ direction [Fig. 3(a) insets, see Appendix A for imaging details]. We have $\overline{\mathrm{OD}}_{s}=\xi \times \overline{\mathrm{OD}}_{x}$ with $\xi \approx 0.8$ to account for the ratio of optical depth integrated along the $\mathbf{k}_{s}$ and $\mathbf{e}_{x}$ directions, respectively. By comparing the quadratic fit that gives $i_{\max , s} \approx$ $4 \times 10^{-4} N \Gamma_{D 2} \overline{\mathrm{OD}}_{x} / 4$ in Fig. 3(b) with Eqs (4) and (6), we find a time-integrated probe Rabi frequency of $\theta_{p} \approx 2 \times 10^{-2}$, taking our best understanding of the efficiency $f_{d}=0.7$ (see Sec. IIIE). This value of $\theta_{p}$ is consistent with the expected excitation by the probe (with peak saturation parameter $s \sim 1$ and duration $\tau_{p}=5 \mathrm{~ns}$ ) in these measurements [53], considering the large uncertainty in the absolute intensity parameter estimations.

We now discuss the enhanced decay rate $\Gamma_{N}$ of the collective emission, which is approximated in Eqs. (4) and (6) under the assumption of negligible angular-dependent emission dynamics (Appendix B). This approximate decay rate corresponds to that of the timed Dicke state [13,16,17,25]. Similar to previous studies of forward superradiance [10,11], we find $\Gamma_{N} \propto N$ for the redirected superradiance, as expected. Here, to make a precise comparison with the theoretical picture, we plot the same data in Fig. 3(b) versus the in situ measured average optical depth $\overline{\mathrm{OD}}_{x}$. From Fig. 3(b), we have $\Gamma_{N} / \Gamma_{D 2} \approx 1.1+0.26 \overline{\mathrm{OD}}_{x}$. Using Eq. (6) again with $\xi=$ $\overline{\mathrm{OD}}_{s} / \overline{\mathrm{OD}}_{x} \approx 0.8$ and the remaining fraction of atoms $(1-$ $l) \approx 0.9$ in these measurements, as discussed in Sec. III E, we obtain $\Gamma_{N} \approx\left(1.1+v \times(1-l) \times \overline{\mathrm{OD}}_{s}\right) \Gamma_{D 2}$ with $v=0.35 \pm$ 0.1 , with no freely adjustable parameters but with an uncertainty limited by the $\overline{\mathrm{OD}}_{s}$ estimation in this paper. The likely discrepancy between this result and the $v=0.25, \Gamma_{N} / \Gamma=$ $1+\overline{\mathrm{OD}} / 4$ prediction of the collective decay of the timed Dicke state $[10,11,13]$ can be expected, since the measured collective emission $i_{s}(t)$ is integrated over the $\sigma$-limited solid angle $\sim 1 /\left(k_{p} \sigma\right)^{2}$ beyond the "exact" $\mathbf{k}_{s}=\mathbf{k}_{p}-2 \mathbf{k}_{c}$ phasematching condition, while the small angle scattering of $\mathbf{E}_{s}\left(\mathbf{k}_{s}\right)$ by the sample itself generally affects the collective emission dynamics [52], thereby violating our assumptions to reach Eq. (4).

\section{Reversible shift of spin-wave $k$ vector}

In the previous section, we have demonstrated the spinwave control for the redirection operation, where a highfidelity spin-wave vector shift $\mathbf{k}_{p} \rightarrow \mathbf{k}_{p}-2 \mathbf{k}_{c}$ is implemented for the background-free detection of superradiance. We proceed further with our geometric control of the spin wave by applying a second pair of switch-off $\left(\mathbf{k}_{p}-2 \mathbf{k}_{c} \rightarrow \mathbf{k}_{p}-4 \mathbf{k}_{c}\right)$ control pulses along the $\mathbf{k}_{c}$ direction, followed by a third control for superradiance recall $\left(\mathbf{k}_{p}-4 \mathbf{k}_{c} \rightarrow \mathbf{k}_{p}-2 \mathbf{k}_{c}\right)$. The superradiant emission $i_{\mathbf{k}_{s}}$ in the $\mathbf{k}_{s}=\mathbf{k}_{p}-2 \mathbf{k}_{c}$ direction is recorded by the photon counter during the full redirectionswitch off-recall sequence. We refer readers to Ref. [30] for discussions of the control sequence and the associated superradiance measurements. Here we provide an example signal $i_{\mathbf{k}_{s}}$ in Fig. 4(ii), where the photon counts are initiated, shut off, and recalled at the expected instances when the three spin-wave control operations as in Fig. 1 are performed.

To understand the observations in Fig. 4, we should note that after the switch-off control, the new wave vector $\mathbf{k}_{s}^{\prime}=$ $\mathbf{k}_{p}-4 \mathbf{k}_{c}$ is associated with wave number $\left|\mathbf{k}_{s}^{\prime}\right| \approx 2.9 \omega_{e g} / c$ that is strongly mismatched from radiation. For our dilute ensemble, this spin-wave excitation should decay in a superradiant-free fashion with approximately the single-atom decay rate $\sim \Gamma_{D 2}$ [37]. At the same time, the emission into the 


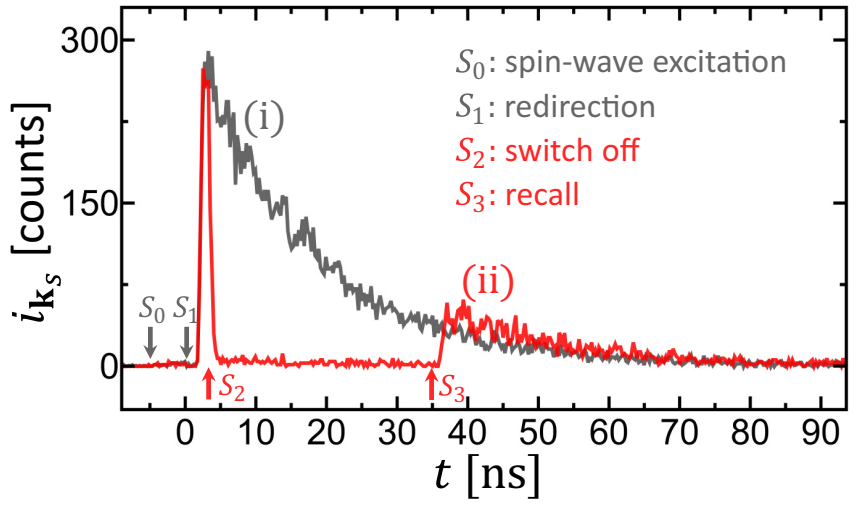

FIG. 4. Experimentally measured photon emission counts $i_{\mathbf{k}_{s}}$ during spin-wave controls. In curve (ii), the collective spontaneous emission along $\mathbf{k}_{s}$ is switched on at $\Delta t_{1}=0.2 \mathrm{~ns}$, switched off at $\Delta t_{2}=0.8 \mathrm{~ns}$, and recalled at $\Delta t_{3}=30.0 \mathrm{~ns}$. The pulses are optimized with $\tau_{c}=0.5 \mathrm{~ns}, \tau_{d}=1.2 \mathrm{~ns}, \Omega_{0} \approx 2 \pi \times 4 \mathrm{GHz}$ and $\delta_{0} \approx 2 \pi \times 4 \mathrm{GHz}$. The spin-wave generation is implemented with a $\tau_{p}=5 \mathrm{~ns}$ probe pulse. Here curve (i) shows the measured $i_{\mathbf{k}_{s}}$ under the same experimental conditions as those for the curve (ii), but for the redirection only and with the switch off and the recall operation removed.

same detection solid angle, without the collective enhancement, should decrease by a factor of $\sim N$ [13]. Following the recall control, the spin wave is phase matched again and the superradiance is recalled at the desired time after a $\Delta t_{3}$ delay. It should be noted that the recalled superradiance decays almost on the same timescale of $\tau \approx 15 \mathrm{~ns}$ as those for the directly redirected superradiance, as shown in reference curve (i) under the otherwise identical experimental conditions. The reduced amplitude of the recalled emission (see Sec. II C) reflects gradual decay of the mismatched $S^{+}\left(\mathbf{k}_{s}^{\prime}\right)$ spin-wave order in the atomic gas (with an experimentally estimated lifetime $\sim 26 \mathrm{~ns}$ in Ref. [30]), before its conversion back to the phase-matched $S^{+}\left(\mathbf{k}_{s}\right)$ excitations. The fact that we can map the phase-mismatched spin-wave order to light should have significant consequences when this technique is applied to arrays, where the subradiant dynamics has been predicted to be particularly rich [24-28].

\section{Optical acceleration}

As discussed in Sec. II A, the control pulse sequence to shift the spin waves also results in a spin-dependent kick, which optically accelerates the phase-patterned $|g\rangle$ states by the geometric force [54]. The momentum transfer along the control beam along $\mathbf{e}_{z}$ can be evaluated by integrating $\left\langle\hat{F}_{z}\right\rangle$ with the single-atom force operator $\hat{F}_{z}=-\frac{\hbar}{2} \partial_{z} \Omega_{c}|a\rangle\langle g|+$ H.c., as the projected atomic state evolves on the $\{|g\rangle-|a\rangle\}$ Bloch sphere [Fig. 1(b)]. For ideal population inversions, the integrated Berry curvature [55] gives the exact photon recoil momentum $\Delta P=2 \hbar k_{c}$ with $\hbar$ the reduced Planck's constant.

Going beyond Ref. [30], we experimentally measure the recoil momentum transfer $\Delta P$ associated with the $D 1$ chirped pulse pair along $\mathbf{e}_{z}$ for the spin wave control, using a time-offlight (TOF) absorption imaging method [Fig. 5(b)]. Keeping in mind the Doppler effects due to the acceleration affect negligibly the nanosecond control dynamics, we repeat a $\mathbf{k} \rightarrow$ (a)
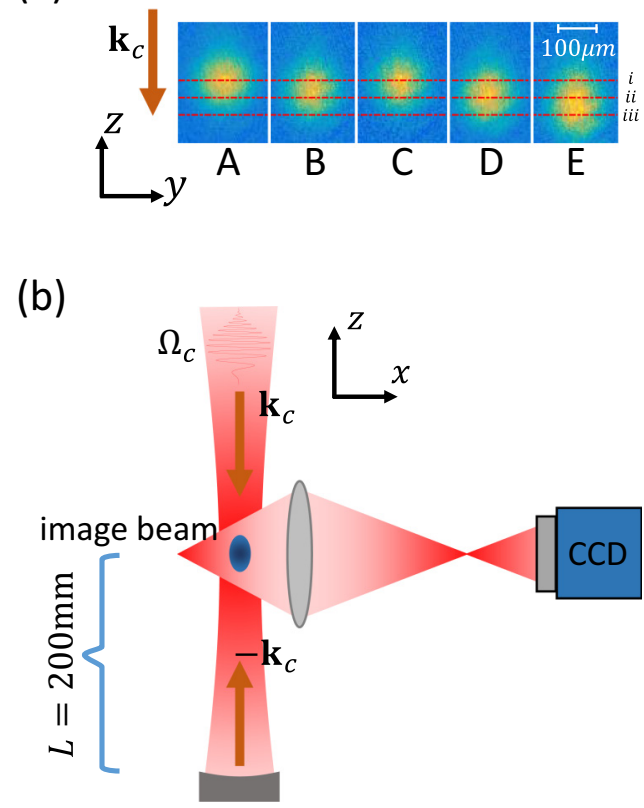

FIG. 5. (a) Typical absorption images of $D 1$ controlled atomic samples after a $T_{\text {tof }}=400 \mu$ s time of flight. The three red dashed lines mark the expected positions for different momentum transfer: $\Delta P=0 \hbar k_{c}(\mathrm{i}), 2 \hbar k_{c}(\mathrm{ii})$, and $4 \hbar k_{c}$ (iii). For images $\mathrm{A}-\mathrm{D}$, each $D 1$ control consists of a pair of chirp pulses with calibrated peak Rabi frequency $\Omega_{0}=0 \mathrm{GHz}, 2 \pi \times 0.8 \mathrm{GHz}, 2 \pi \times 1.9 \mathrm{GHz}$, and $2 \pi \times 2.7 \mathrm{GHz}$ and chirp parameter $\delta_{0}=2 \pi \times 0.1 \mathrm{GHz}, 2 \pi \times 0.1$ $\mathrm{GHz}, 2 \pi \times 0.1 \mathrm{GHz}$, and $2 \pi \times 3.4 \mathrm{GHz}$, respectively. For image E, each $D 1$ control consists of two pairs of chirped pulses with the same parameters as that for image D. The central positions of the atomic samples can be obtained with Gaussian fits. These parameter combinations are also marked in Fig. 6(a). (b) Imaging setup for the optical acceleration measurements. The setup is with aberration-free numerical aperture of $\mathrm{NA} \approx 0.3$.

$\mathbf{k}-2 \mathbf{k}_{c}$ control pulse $N_{\text {rep }}=5$ times to enhance the measurement sensitivity. The period $T_{\text {rep }}=440 \mathrm{~ns} \gg 1 / \Gamma_{D 1}$ is set to ensure independent interactions. We then measure the central position shift of the atomic sample after a $T_{\text {tof }}=400 \mu \mathrm{s}$ TOF, using calibrated absorption images. For the absorption imaging, the atomic sample is illuminated by a $\tau_{\exp }=20 \mu \mathrm{s}$ imaging pulse resonant to $5 S_{1 / 2} F=2-5 P_{3 / 2} F^{\prime}=3$ along the $\mathbf{e}_{x}$ direction. The $2 \mathrm{D}$ transmission profile $T(y, z)=I / I_{0}$ is obtained by processing the imaging beam intensity $I, I_{0}$ on a CCD camera with and without the atomic sample, respectively. We then process $T(y, z)$ to obtain the optical depth profile $\operatorname{OD}(y, z)$ of the samples (also see Appendix A). Next, the central position shifts $\Delta z_{0}$ are obtained with 2D Gaussian fits for samples with and without the control pulses. Finally, a velocity change is estimated as $\Delta v=\Delta z_{0} /\left(T_{\text {tof }}+\tau_{\exp } / 2\right)$. Typical absorption images are presented in Fig. 5(a) for samples under $D 1$ control pulses with various $\Omega_{0}$ and $\delta_{0}$ parameters as defined in Sec. III A. In particular, we expect that the parameters used in image D in Fig. 5(a) lie in an error-resilient region of parameter space and reflect the nearly ideal momentum change of $\Delta P=2 \hbar k_{c}$. We shortly show that the measured momentum kicks agree well with numerical models. 
(a)

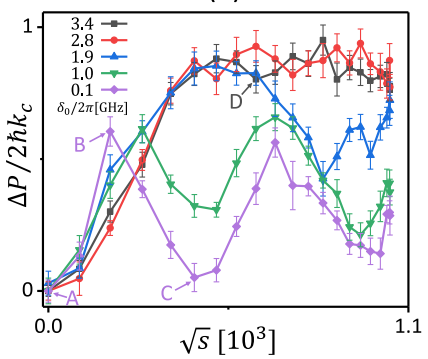

(b)

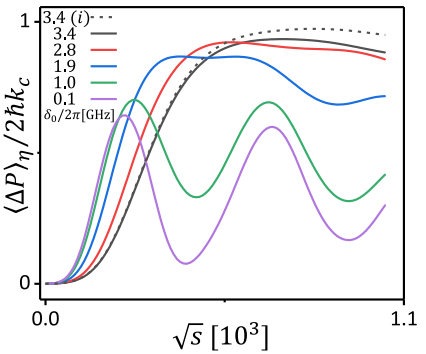

(c)

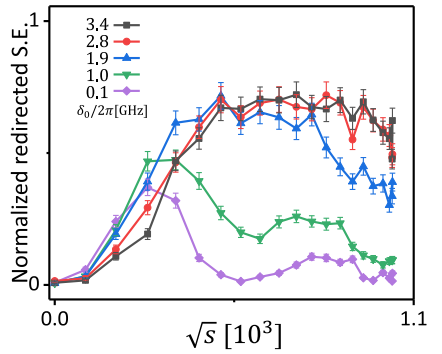

(d)

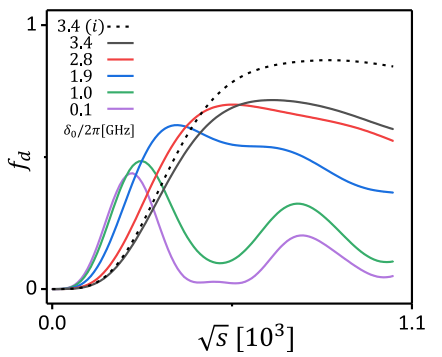

FIG. 6. Optical acceleration and efficiency for a $\mathbf{k} \rightarrow \mathbf{k}-2 \mathbf{k}_{c}$ spin-wave control with various pulse shaping parameters. Here $\tau_{c}=$ $0.9 \mathrm{~ns}, \tau_{d}=1.36 \mathrm{~ns}$. The mean momentum shift $\Delta P$ in (a) and normalized redirected superradiance (S.E.) in (c) are plotted versus peak laser intensity parameter $\sqrt{s}$ for control pulses with various chirping parameters $\delta_{0}$. (b) and (d) are simulated momentum transfers and dipole control efficiencies $f_{d}$ with additional laser pulse parameters optimally estimated. The simulation also provides acceleration and dipole control efficiency for an atom starting in the Zeeman state $m_{F}=0$ (dashed lines labeled with (i) in the legends). In (a), the A-D markers give parameters for absorption images presented in Fig. 5 .

With the knowledge of recoil velocity $v_{r}=\hbar k_{c} / m \approx$ $5.8 \mathrm{~mm} / \mathrm{s}$, we obtain $\Delta P=\Delta v / N_{\text {rep }} v_{r} \times \hbar k_{c}$ per $D 1$ control. Typical $\Delta P$ measurements are presented in Fig. 6(a) versus intensity parameter $\sqrt{s}$, for shaped pulses with different chirping parameters $\delta_{0}$. For controls with nearly zero chirp $\left(\delta_{0}=\right.$ $2 \pi \times 0.1,1.0 \mathrm{GHz}), \Delta P$ displays a damped oscillation, which is due to optical Rabi oscillations with broadened periodicity associated with intensity inhomogeneity of the focused laser. The oscillation is suppressed at large $\delta_{0}$, with $\Delta P$ reaching $89(4) \%$ of the $\sim 2 \hbar k_{c}$ limit at large $s$, suggesting a robustness to our coherent control process. Similar measurements are performed for the reversed $\mathbf{k} \rightarrow \mathbf{k}+2 \mathbf{k}_{c}$ controls which results in opposite momentum shifts.

\section{E. Control efficiency: calibration and optimization}

To quantify the imperfections in implementing the spin-wave control by geometric phase patterning $\left[U_{g}\right.$ in Eq. (2)], we need to properly model the dissipative dynamics of collective dipoles. For this purpose, we introduce the coherent dipole control efficiency, $f_{d}=$ $\left\langle\operatorname{tr}\left(\rho_{\eta} S^{+}\left(\mathbf{k}_{s}\right) S^{-}\left(\mathbf{k}_{s}\right)\right)\right\rangle_{\eta} / \operatorname{tr}\left(\rho_{0} S^{+}\left(\mathbf{k}_{s}\right) S^{-}\left(\mathbf{k}_{s}\right)\right)$, with $\rho_{\eta}, \rho_{0}$ the density matrix that describes the weakly $D 2$ excited atomic sample subjected to the nonideal $\tilde{U}_{g}$ and the ideal, instantaneous $U_{g}(\Delta \mathbf{k})$ control by Eq. (2), respectively. Here $\tilde{U}_{g}\left(\Delta \mathbf{k} ; \Omega_{0}, \delta_{0}, \eta\right)$ due to the nanosecond shaped pulse control is parameterized by the peak Rabi frequency $\Omega_{0}$ and chirping parameter $\delta_{0}$, as well as factors $\eta_{1,2}$ as the normalized laser intensities for the forward and retroreflected pulses locally seen by the atoms. The control efficiency $f_{d}$ is averaged over the Gaussian intensity distribution.

To optimize the $\mathbf{k} \rightarrow \mathbf{k}-2 \mathbf{k}_{c}$ shift by the nonideal $\tilde{U}_{g}\left(2 \mathbf{k}_{c} ; \Omega_{0}, \delta_{0}, \eta\right)$ control, experimentally we simply scan the control pulse shaping parameters $\sqrt{s} \propto \Omega_{0}$ and $\delta_{0}$ to maximize the redirected superradiant emission that generate the time-dependent signal such as the curve (i) in Fig. 4. The data in Fig. 6(c) are the corresponding total photon counts by integrating the time-dependent signals. By optimizing the total counts, we are able to locate the optimal pulse shaping parameters $\Omega_{0}=2 \pi \times 2.7 \mathrm{GHz}$ and $\delta_{0}=2 \pi \times 3.4 \mathrm{GHz}$ for the $\tau_{c}=0.9 \mathrm{~ns}$ chirped-sine pulses in these experiments.
The magnitude of the optimally redirected superradiant emission scales quadratically with total atom number $N$ and increases with both the probe excitation strength $\left|\Omega_{p} \tau_{p}\right|^{2} \ll 1$ and the control efficiency $f_{d}$ [see Eqs. (4) and (6)]. However, without accurate knowledge of the experimental parameters associated with the spin-wave preparation and emission detection, it is difficult to precisely quantify $f_{d}$ with the photon counting readouts. Instead, we calibrate the spin-wave control efficiency $f_{d}$ with a numerical modeling strategy. In particular, we perform density-matrix calculations of a single atom interacting with the control pulses, including full hyperfine structure. Both the collective spin wave shifts and matter-wave acceleration can be evaluated from the singleatom results, if atom-atom interactions and re-scattering of the control fields is negligible, as we expect to be the case for the low atomic densities and high pulse bandwidths used in our work. For the calibration, we first adjust experimental parameters in numerical simulations so as to optimally match the simulated average momentum shift $\langle\Delta P\rangle_{\eta}$ in Fig. 6(b) with the absolute experimental measurements in Fig. 6(a). The corresponding $f_{d}$ under identical experimental conditions are then calculated as in Fig. 6(d). The fairly nice match between the superradiance measurements in Fig. 6(c) and Fig. 6(d) is achieved by uniformly normalizing the total counts in Fig. 6(c), with no additionally adjusted parameters. Near the optimal control regime, the simulation suggests we have reached a collective dipole control efficiency $f_{d}=72 \pm 4 \%$, accompanied with the observed $f_{a} \equiv \Delta P / 2 \hbar k_{c}=89 \pm 4 \%$ acceleration efficiency. Constrained by the absolute acceleration measurements, we found this optimal $f_{d}$ estimation to be quite robust in numerical modeling when small pulse-shaping imperfections are introduced.

On the other hand, for a full redirection-switch off-recall sequence as in Fig. 4, we can also estimate the efficiency of the $\mathbf{k} \rightarrow \mathbf{k}+2 \mathbf{k}_{c}$ recall. We fit the amplitude of the recalled superradiant emission in the short $\Delta t_{3}$ limit, and compare that with the amplitude of the redirected emission with the same experimental sequence [30]. The ratio between the two fluorescence signal amplitudes defines an overall storage-recall efficiency for the controlled dipole spin wave intensity of $\sim 58 \%$. By assuming equal efficiency for each of the two operations, the efficiency for a single $\mathbf{k} \rightarrow \mathbf{k} \pm 2 \mathbf{k}_{c}$ shift is 
thus at the $75(5) \%(\sim \sqrt{58 \%})$ level, which are performed using the second type of delay line [Fig. 2(b)] and reoptimized pulse-shaping parameters. The efficiency is also consistent with the prediction by numerical modelings, as discussed in Appendix B.

The optimal $f_{d}$ as in Fig. 6(c) is limited by $m_{F}$-dependent hyperfine phase shifts and $D 1+D 2$ spontaneous decays during the $\tau_{d}+\tau_{c}=2.26$ ns control. In particular, a $l \sim 10 \%$ atom loss due to $D 1$ spontaneous emission and $5 P_{1 / 2}$ population trapping (particularly for $\left|m_{F}\right|=1$ states) is expected to reduce the number of atoms participating in the $D 2 \mathrm{col}-$ lective emission. With atoms prepared in a single $m_{F}=0$ state, spontaneous emission limited dipole control efficiency of $f_{d} \approx 87 \%$, accompanied with an acceleration efficiency $f_{a} \approx 97 \%$ should be reachable [Figs. 6(b) and 6(d)] with the same control pulses.

\section{DISCUSSIONS}

The error-resilient state-dependent phase patterning technique demonstrated in this paper is a general method to precisely control dipole spin waves in atomic gases and the associated highly directional collective spontaneous emission in the time domain [37,56-58]. The control is itself a single-body technique, which can be accurately modeled for dilute atomic gases when the competing resonant dipole-dipole interactions between atoms can be ignored during the pulse duration. With the geometric phase inherited from the optical phases of the control laser beams, it is straightforward to design $\varphi_{\mathrm{G}}$ beyond the linear phase used in this work and to manipulate the collective spin excitation in complex ways tailored by the control beam wavefronts.

We note that the atomic motion associated with the control can limit the coherence time of the spin-wave order in free gases at finite temperature, and that the limiting effect can be suppressed with optical lattice confinements [30]. In the following, we discuss methods for perfecting the spin-wave control in dense gases and then summarize possible prospects opened by this work.

\section{A. Toward perfect control with pulse shaping}

The optical dipole spin-wave control in this work is subjected to various imperfections at the single-body level. The pulse-shaping errors combined with laser intensity variations lead to imperfect population inversions and reduced operation fidelity. The imbalanced beam pair intensities lead to spatially dependent residual dynamical phase writing and distortion of the collective emission mode profiles. The hyperfine coupling of the electronically excited states may lead to inhomogeneous phase broadening as well as hyperfine Raman couplings, resulting in coherence and population losses as in this work. Finally, the spontaneous decays on both the $D 1$ control and $D 2$ probe channels limit the efficiency of the finite-duration pulse control. However, the imperfections of the control stemming from the single-atom effects are generally manageable with better quantum control techniques [59-62] well-developed in other fields, if they can be implemented in the optical domain with reliable pulse-shaping systems of sufficient precision, bandwidth, and output power.
Beyond single-body effects, we emphasize that with increased $\Omega_{c}$ strength and reduced $\tau_{c, d}$ time, it is generally possible to suppress interaction effects so as to maintain the precision enabled by the single-body simplicity, for precise spin-wave control in denser atomic gases.

As discussed in Sec. IIIE, the pulse-shaping system used in this work already supports $f_{d} \sim 87 \%$ efficiency if atomic $m_{F}$ states are better prepared, which is then limited by the $D 1$ and $D 2$ spontaneous decay (single-atom limit) during the $\tau_{c}+\tau_{d}=2.26 \mathrm{~ns}$ control time. Instead of imparting geometric phase to the ground-state atoms, in future work an $|e\rangle-|a\rangle$ transition with a longer $|a\rangle$ lifetime [63] may be chosen to implement a $U_{e}\left(\varphi_{\mathrm{G}}\right)$ for $|e\rangle$-state phase-patterning. The influence from the $D 1$ decay can thus be eliminated, leading to $f_{d} \sim 95 \%$ limited by the suppressed $D 2$ decay. With an additional fivefold reduction of $\tau_{c}$ to enable $\tau_{c}+\tau_{d}$ to $\sim 400 \mathrm{ps}$, aided by the well-developed advanced error-resilient techniques [59-62], we expect $f_{d}$ reaching $99 \%$ for highfidelity dipole spin-wave control.

For the error-resilient shaped optical pulse control, ideally the fivefold reduction of control time from the $\tau_{c}=0.5-0.9 \mathrm{~ns}$ pulses in this work needs to be supported by a fivefold increase of laser modulation bandwidth and a 25 -fold increase of laser intensity. Starting from the subnanosecond pulse-shaping technique in this paper detailed in Ref. [30], the improvement may be achieved with a combined effort of stronger input, wider modulation, and tighter laser focus. As a promising alternative, the control pulses may also be generated with mode-locked lasers [34,35,64-67] with orders of magnitudes enhanced peak power and pulse bandwidth. Here we notice that for the same control operation, the required pulse peak power and energy scales with $1 / \tau_{c}^{2}$ and $1 / \tau_{c}$, respectively. For controlling macroscopic samples as in this work, the scaling toward ultrafast pulses can become demanding enough to require sophisticated optical pulse amplifications, compromising the setup flexibility. In addition, the control strength $\Omega_{c} \gg 1 / \tau_{c}$ and the modulation bandwidth are also upper-bounded to minimize uncontrolled light shifts and multiphoton excitations. Therefore, for the purpose of precisely and flexibly controlling dipole spin waves, it appears shaping picosecond pulses is more preferred than shaping ultrafast pulses $[34,64,68,69]$ for generating nearly resonant pulses with a suitable duration and modulation bandwidth.

\section{B. Summary and outlook}

In this paper, we experimentally demonstrate and systematically study a state-dependent geometric phase patterning technique for control of collective spontaneous emission by precisely shifting the $\mathbf{k}$ vector of dipole spin waves in the time domain. The method involves precisely imparting geometric phases to electric dipoles in a large sample, using a focused laser beam with large intensity inhomogeneities. Similar error-resilient techniques have been widely applied in nuclear magnetic resonance $[44,61,62,70]$. Our work represents a step in exploring such error resilience toward optical control of dipole spin waves near the unitary limit and for efficient far-field access to the rarely explored phase-mismatched optical spin-wave states. During the characterization of our method, we also made intriguing observations related to fun- 
damental properties of spin-wave excitations. These include a verification of the $i_{N} \propto N^{2}$ scaling law, a qualification of the enhancement relation $\Gamma_{N} / \Gamma=1+\overline{\mathrm{OD}} / 4$, and an observation of matter-wave acceleration accompanying the spin-wave control. We have provided a theoretical analysis of this spin wave and spontaneous emission control. Instead of working with free-space and randomly positioned atoms, our control technique can be readily applied to atomic arrays for efficient access to highly subradiant states. The technique may open the door to related applications envisaged in the field of quantum optics [37,71], to help unlock nontrivial physics of long-lived and interacting dipole spin-waves in dense atomic gases [24-29,50,72-75], and to enable nonlinear quantum optics based on subradiance-assisted resonant dipole interaction [76-78].

Finally, on the laser technology side, we hope this paper motivates additional developments of continuous and ultrafast pulse-shaping methods for optimal quantum control of optical electric dipoles.

\section{ACKNOWLEDGMENTS}

We are grateful to Prof. Lei Zhou for both helpful discussions and for kind support and to Prof. J. V. Porto and Prof. Da-Wei Wang for helpful discussions and insightful comments on the paper. We thank Prof. Kai-Feng Zhao and Prof. Zheng-Hua An for help on developing pulseshaping technology and for support from the Fudan Physics nanofabrication center. D.E.C. acknowledges support from the European Union's Horizon 2020 research and innovation program under European Research Council Grant Agreement No. 639643 (FOQAL) and Grant Agreement No. 899275 (DAALI), MINECO Severo Ochoa Program No. CEX2019000910-S, Generalitat de Catalunya through the CERCA program and QuantumCat (Reference No. 001-P-001644), Fundacio Privada Cellex, Fundacio Mir-Puig, Fundacion Ramon Areces Project CODEC, and Plan Nacional Grant ALIQS funded by Ministerio de Ciencia, Innovación y Universidades, Agencia Estatal de Investigación, and European Regional Development Fund. This research is mainly supported by National Key Research Program of China under Grants No. 2016YFA0302000 and No. 2017YFA0304204, by NSFC under Grant No. 11574053, and by Shanghai Scientific Research Program under Grant No. 15ZR1403200.

\section{APPENDIX A: EXPERIMENTAL DETAILS}

\section{Resonant OD and atom number measurements}

The absorption imaging setup as schematically illustrated in Fig. 5 not only helps us to quantify the optical acceleration effect with TOF technique, but also to directly measure the optical depth profile $\operatorname{OD}_{x}(y, z)$ and atom number $N$ as in Sec. III B. To investigate the $\Gamma_{N} / \Gamma=1+\overline{\mathrm{OD}} / 4$ relation, extra care was taken to extract the $\operatorname{OD}_{x}(y, z)$ images from the resonant absorption images. Here $\operatorname{OD}_{x}(y, z)$ to be measured should be the unpolarized atoms in the weak excitation limit, with in situ $\varrho(\mathbf{r})$ distribution close to those in the quantum optics experiments and for both low $\overline{\mathrm{OD}}<1$ and quite high $\overline{\mathrm{OD}} \sim 3.5$. To ensure consistent $\varrho(\mathbf{r})$ distribution to be measured, a short exposure time of $20 \mu$ s is chosen. To collect sufficient counts on the camera, we use imaging beams with quite high intensity in the range of $I_{0}=1 \sim 20 \mathrm{~mW} / \mathrm{cm}^{2}$ and thus with a saturation parameter $s=0.3 \sim 7$ assuming $I_{s}=3.05 \mathrm{~mW} / \mathrm{cm}^{2}$ [51] for $\pi$ transition of $5 S_{1 / 2} F=$ $2-5 P_{3 / 2} F^{\prime}=3$. We reduce the measurement uncertainty related to saturation effects following techniques similar to Refs. [79,80]. In addition, to avoid measurement uncertainty related to low local counts for the highly absorbing samples, we calibrate the peak OD of the in situ samples with TOF images at reduced OD. The processes are detailed as follows.

We start by repeated absorption imaging measurements for nearly identical TOF samples with $2 \mathrm{D}$ transmission profile $T(I)=I / I_{0}>75 \%$, with incoming $I_{0}(y, z)$ and transmitted $I(y, z)$ intensities recorded on the camera. The optical depth profile in the weak excitation limit can be approximately as $\mathrm{OD}_{x}(y, z)=-\log T(I)+\left(I_{0}-I\right) / I_{s}^{\text {eff }}[79,80]$. Here $I_{s}^{\text {eff }}$ is an effective parameter for calibrating our saturation intensity measurements. By globally adjusting $I_{s}^{\text {eff }}$ and thus the $\left(I_{0}-I\right) / I_{s}^{\text {eff }}$ term, we obtain consistent $\operatorname{OD}_{x}(y, z)$ from all the measurements with $I_{0}=1 \sim 20 \mathrm{~mW} / \mathrm{cm}^{2}$ with minimal variations. Notice that the radiation pressure during the imaging process does not significantly vary the power-broadened atomic response.

The optimally adjusted $I_{s}^{\text {eff }}$ serves to extract the $\operatorname{OD}_{x}(y, z)$ spatial profile for atomic sample immediately after their release from the dipole trap, as in Fig. 3(a) with approximately identical spatial profiles. In addition, under the consistent atomic sample preparation conditions, we also measure the optical depth profile $\operatorname{OD}_{x}^{\prime}(y, z)$ and total atom number after a $430 \mu \mathrm{s}$ TOF. The TOF greatly reduces the peak linear absorption for the highest OD sample here from the expected $95 \% \sim 99 \%$ level down to $15 \% \sim 25 \%$, leading to more accurate estimation of integrated OD that is served to calibrate the in situ $\overline{\mathrm{OD}}_{x}$ measurements. To account for optical pumping effects that tend to increase the $F=2-F^{\prime}=3$ light-atom coupling strengths, a factor of 0.85 [51] is multiplied to the extracted $\mathrm{OD}_{x}(y, z)$.

We finally adjust $\mathrm{OD}_{x}$ due to the imaging laser frequency noise in this work by up to $30 \%$, according to the measured linewidth broadening of the TOF sample absorption spectrum and then obtain $\overline{\mathrm{OD}}_{s}$ using the sample aspect ratio estimated by the auxiliary imaging optics along $\mathbf{e}_{z}$. These last two steps introduce the largest uncertainties into our $\overline{\mathrm{OD}}_{s}$ estimation. It is worth noting that the laser noise correction tends to reduce the $v$ value in Sec. III B. We use $\sigma_{r}=1.59 \times 10^{-9} \mathrm{~cm}^{2}$ for linearly polarized probe on $5 S_{1 / 2}, F=2$ levels to estimate $N=\frac{1}{\sigma_{r}} \int \mathrm{OD}_{x}(y, z) d y d z$.

\section{APPENDIX B: THEORETICAL MODEL AND NUMERICAL SIMULATION}

This Appendix provides a theoretical background associated with the experimental observations for both this paper and Ref. [30]. First, in Appendix B 1, we provide a minimal model to explain the collective spontaneous emission by the controlled spin waves. Next, in light of short control time $\tau_{c, d}$ with negligible atom-atom interaction effects, we set up a single-atom model to explain the control of spin waves supported by the nearly noninteracting atoms in Appendix B2-B4. Different from Ref. [30], here we outline the method to model the interaction between the atomic sample with the 
focused laser beam by estimating the most likely experimental parameters, so as to clearly understand the physical limitations behind the inefficiency of our spin-wave control. Finally, in Appendix B5 we discuss the influence of controlled spin-wave dynamics subject to hyperfine interactions during the superradiant recall operation.

\section{Collective spontaneous emission from a dilute gas of two-level atoms}

We consider the interaction between $N$ two-level atoms with a resonant electromagnetic field at wavelength $\lambda_{p}$ and frequency $\omega_{e g}$. With transition matrix element $\mathbf{d}_{e g}=d_{e g} \mathbf{e}_{d}$, the absorption cross section is given by $\sigma_{r}=k_{p} \alpha_{i}$ with $\alpha_{i}=$ $2\left|\mathbf{d}_{e g}\right|^{2} / \hbar \Gamma, \Gamma$ being the linewidth of the $|e\rangle-|g\rangle$ transition. The atomic ensemble follows an average spatial density distribution $\varrho(\mathbf{r})=\left\langle\sum_{i} \delta\left(\mathbf{r}-\mathbf{r}_{i}\right)\right\rangle$ that is assumed to be nearly spherical and smooth, in particular, $\varrho(\mathbf{r})$ does not vary substantially on length scales other than that close to its characteristic radius $\sigma \gg \lambda_{p}$. We further restrict our discussion to intermediate sample size with $\sigma \ll c \tau$, with $c$ the speed of light and $\tau$ the shortest time-scale of interest. The transmission of a plane-wave resonant probe beam at the exit of the atomic sample, in the $\mathbf{r}=\left\{\mathbf{r}_{\perp}, r_{p}\right\}$ coordinate, follows the Beer-Lambert law with transmission $T\left(\mathbf{r}_{\perp}\right)=e^{-\mathrm{OD}\left(\mathbf{r}_{\perp}\right)}$. The $2 \mathrm{D}$ optical depth distribution is given by $\mathrm{OD}=N \varrho_{c}\left(\mathbf{r}_{\perp}\right) \sigma_{r}$, $\varrho_{c}\left(\mathbf{r}_{\perp}\right)=\frac{1}{N} \int \varrho(\mathbf{r}) d r_{p}$ being the normalized column density.

To describe both the collective dipole dynamics and its collective radiation, we regard the small atomic sample as system and free-space optical modes as reservoir. The electric-dipole interaction can be effectively described by the many-atom density matrix $\rho$, after the photon degrees of freedom are eliminated by the standard Wigner-Weisskopf procedure. Following the general approach [24,81], the density matrix $\rho$ obeys the master equation $\dot{\rho}=\frac{1}{i \hbar}\left(H_{\text {eff }} \rho-\rho H_{\text {eff }}^{\dagger}\right)+L_{c}[\rho]$, where $L_{c}$ is the population recycling superoperator associated with random quantum jumps in the stochastic wave-function picture. Here we focus on the effective Hamiltonian $H_{\text {eff }}$ that governs the deterministic evolution of states and observables. The non-Hermitian effective Hamiltonian can be expressed as

$$
H_{\mathrm{eff}}=\sum_{i} H_{a}^{i}+\hat{V}_{\mathrm{DD}, \mathrm{eff}},
$$

with single-atom Hamiltonian $H_{a}^{i}$ for atom at location $\mathbf{r}_{i}$, and effective dipole-dipole interaction operator $\hat{V}_{\mathrm{DD} \text {, eff }}=$ $\sum_{i, j} \hat{V}_{\mathrm{DD}}^{i, j}$ that sums over the pairwise resonant dipole interaction:

$$
\hat{V}_{\mathrm{DD}}^{i, j}=-\frac{k_{p}^{2}}{\varepsilon_{0}} \mathbf{d}_{e g}^{*} \cdot \mathbf{G}\left(\mathbf{r}_{i}-\mathbf{r}_{j}, \omega_{e g}\right) \cdot \mathbf{d}_{e g} \sigma_{i}^{+} \sigma_{j}^{-} .
$$

Here $\sigma_{i}^{+}=\left|e_{i}\right\rangle\left\langle g_{i}\right|, \sigma_{i}^{-}=\left(\sigma_{i}^{+}\right)^{\dagger}$ are the raising and lowering operators for the $i^{\text {th }}$ atom and $\varepsilon_{0}$ is the vacuum permittivity. $\mathbf{G}\left(\mathbf{r}, \omega_{e g}\right)$ is the free-space Green's tensor of the electric field obeying

$$
\nabla \times \nabla \times \mathbf{G}\left(\mathbf{r}, \omega_{e g}\right)-\frac{\omega_{e g}^{2}}{c^{2}} \mathbf{G}\left(\mathbf{r}, \omega_{e g}\right)=\delta^{3}(\mathbf{r}) \mathbb{1} .
$$

Intuitively, Eq. (B2) allows for the exchange of excitations between atoms, which is mediated by photon emission and reabsorption, and whose amplitude thus naturally depends on $\mathbf{G}\left(\mathbf{r}, \omega_{e g}\right)$ which describes how light propagates from one atomic position to another.

With the spin-model description of the atomic dipole degrees of freedom, the electric field operator, describing the light emitted by the atoms, can be written in terms of the atomic properties as

$$
\hat{\mathbf{E}}_{s}(\mathbf{r})=\frac{k_{p}^{2}}{\varepsilon_{0}} \sum_{i}^{N} \mathbf{G}\left(\mathbf{r}-\mathbf{r}_{i}, \omega_{e g}\right) \cdot \mathbf{d}_{e g} \sigma_{i}^{-} .
$$

Instead of generally discussing evolution of atomic states in the $N$-spin space governed by $H_{\text {eff }}$, in the following we discuss the timed-Dicke state $\left|\psi_{\mathrm{TD}}(\mathbf{k})\right\rangle=S^{+}(\mathbf{k})\left|g_{1}, g_{2}, \ldots, g_{N}\right\rangle$ and observables composed of collective linear operators. The results can then be straightforwardly applied to weakly excited gases in the linear optics regime as in this experiment.

We first consider the field amplitude of the spontaneously emitted photons. The emitted single photon from a timed Dicke state has a spatial mode profile $\boldsymbol{\varepsilon}_{\mathbf{k}}(\mathbf{r})=$ $\left\langle g_{1}, g_{2}, \ldots, g_{N}\left|\hat{\mathbf{E}}_{s}(\mathbf{r})\right| \psi_{\mathrm{TD}}(\mathbf{k})\right\rangle$, which is readily rewritten after the $\left\{\mathbf{r}_{i}\right\}$-configuration average as

$$
\overline{\boldsymbol{\varepsilon}_{\mathbf{k}}}(\mathbf{r})=\frac{k_{p}^{2} \sqrt{N}}{\varepsilon_{0}} \int \mathbf{G}\left(\mathbf{r}-\mathbf{r}^{\prime}, \omega_{e g}\right) \cdot \mathbf{d}_{e g} e^{i \mathbf{k} \cdot \mathbf{r}^{\prime}} \varrho\left(\mathbf{r}^{\prime}\right) d^{3} r^{\prime} .
$$

Writing the spatial coordinate as $r=\left\{r_{\perp}, r_{\mathbf{k}}\right\}$, one can first integrate Eq. (B5) at a fixed perpendicular coordinate over $r_{p}$, to obtain the emitted field at the end of the sample as in Eq. (3) with $\delta k=|\mathbf{k}|-\omega_{e g} / c$. The approximate integration assumes slowly varying amplitude along both $\mathbf{r}_{\perp}$ and $r_{\mathbf{k}}$ directions. For $\mathbf{k}=\mathbf{k}_{p}$ with $\delta k=0$, we then integrate the corresponding intensity over all transverse positions $\mathbf{r}_{\perp}$, and normalize the radiation power by the energy $\hbar \omega_{e g}$ of a single photon to obtain the collective photon emission rate $i_{\mathbf{k}_{p}}^{(1)}=$ $\frac{2 \varepsilon_{0} c}{\hbar \omega_{e g}} \int\left|\overline{\boldsymbol{\varepsilon}_{p}}(\mathbf{r})\right|^{2} d^{2} \mathbf{r}_{\perp} \approx \overline{\mathrm{OD}}_{p} \Gamma / 4$. For weakly excited coherent spin-wave excitation, this emission rate is multiplied by $N \theta_{p}^{2}$ as in Eq. (4).

We now discuss time dependence of collective spontaneous emission described by Eq. (4) in the main text. The topic is related to a collective Lamb shift in a dilute atomic gas, an important and quite subtle effect well studied in previous work $[16,82]$. To apply the general theoretical predictions to this paper, we explore the spin model [24] to revisit the decay part of the problem, for the quite dense and small samples here.

We consider free gas evolution with $H_{\text {eff }}=$ $\hat{V}_{\text {DD,eff }}$ and time-dependent field amplitude $\boldsymbol{\varepsilon}_{p}(\mathbf{r}, \mathbf{t})=$ $\left\langle g_{1}, g_{2}, \ldots, g_{N}\left|\hat{\mathbf{E}}_{s}(\mathbf{r}, t)\right| \psi_{\mathrm{TD}}\left(\mathbf{k}_{p}\right)\right\rangle$, for $\left|\mathbf{r}-\mathbf{r}_{i}\right| \gg \lambda_{p}$ and with $\hat{\mathbf{E}}_{s}(\mathbf{r}, t)$ evolving according to Heisenberg-Langevin equation $\dot{\hat{\mathbf{E}}}_{s}=\frac{1}{i \hbar}\left(\hat{\mathbf{E}}_{s} \hat{V}_{\mathrm{DD}, \mathrm{eff}}-\hat{V}_{\mathrm{DD}, \mathrm{eff}}^{\dagger} \hat{\mathbf{E}}_{s}\right)+\hat{f}$. With the Langevin force $\hat{f}$ being averaged to zero, for $|\psi\rangle=\left|\psi_{\mathrm{TD}}\left(\mathbf{k}_{p}\right)\right\rangle$ we have

$\left.\dot{\boldsymbol{\varepsilon}}_{p}(\mathbf{r}, \mathbf{t})=-i\left\langle g_{1}, g_{2}, \ldots, g_{N}\right| \hat{\mathbf{E}}_{s}(\mathbf{r}, t)\right) \hat{V}_{\mathrm{DD}, \mathrm{eff}}\left|\psi_{\mathrm{TD}}\left(\mathbf{k}_{p}\right)\right\rangle$.

To evaluate Eq. (B6), we insert the orthogonal timed-Dicke basis $\left\{\left|\psi_{\mathrm{TD}}\left(\mathbf{k}_{p}\right)\right\rangle,\left|\psi_{1}\left(\mathbf{k}_{p}\right)\right\rangle, \ldots,\left|\psi_{N-1}\left(\mathbf{k}_{p}\right)\right\rangle\right\}$ as in Ref. [16] into the equation. Here $\left|\psi_{n}\left(\mathbf{k}_{p}\right)\right\rangle=S_{n}^{+}\left(\mathbf{k}_{p}\right)\left|g_{1}, \ldots, g_{N}\right\rangle$ are single-excitation collective states with $S_{n}^{+}\left(\mathbf{k}_{p}\right)=\sum_{i} c_{n, i} \sigma_{i}^{+}$, $n=1, \ldots, N-1$ and with $c_{n, i}$ properly chosen to ensure the basis orthogonality [16]. We further define the far-field emission amplitudes associated with the $N-1\left|\psi_{n}\left(\mathbf{k}_{p}\right)\right\rangle$ states 
as $\left.\boldsymbol{\varepsilon}_{n}(\mathbf{r}, t)=\left\langle g_{1}, g_{2}, \ldots, g_{N}\right| \hat{\mathbf{E}}_{s}(\mathbf{r}, t)\right) \mid \psi_{n}\left(\mathbf{k}_{p}\right\rangle$. We have

$$
\begin{aligned}
\dot{\boldsymbol{\varepsilon}}_{p}(\mathbf{r}, t)= & -i V_{\mathrm{DD}}\left(\mathbf{k}_{p}, \mathbf{k}_{p}\right) \boldsymbol{\varepsilon}_{p}(\mathbf{r}, t) \\
& -i \sum_{n} V_{\mathrm{DD}}\left(n, \mathbf{k}_{p}\right) \boldsymbol{\varepsilon}_{n}(\mathbf{r}, t),
\end{aligned}
$$

with $V_{\mathrm{DD}}\left(\mathbf{k}_{p}, \mathbf{k}_{p}\right)=\left\langle\psi_{\mathrm{TD}}\left(\mathbf{k}_{p}\right)\left|\hat{V}_{\mathrm{DD}, \text { eff }}\right| \psi_{\mathrm{TD}}\left(\mathbf{k}_{p}\right)\right\rangle$ and similarly $V_{\mathrm{DD}}\left(n, \mathbf{k}_{p}\right)=\left\langle\psi_{n}\left(\mathbf{k}_{p}\right)\left|\hat{V}_{\mathrm{DD}, \text { eff }}\right| \psi_{\mathrm{TD}}\left(\mathbf{k}_{p}\right)\right\rangle$. The second line of Eq. (B7) includes random and collective couplings between the $\mathbf{k}_{p}$ superradiant excitation and other super- and subradiant modes [83], a fact associated with $\left|\psi_{\mathrm{TD}}\left(\mathbf{k}_{p}\right)\right\rangle$ not being the eigenstate of $\hat{V}_{\mathrm{DD}, \text { eff }}[16,82,84]$.

The $V_{\mathrm{DD}}\left(\mathbf{k}_{p}, \mathbf{k}_{p}\right) \propto \sum_{i, j} \mathbf{d}_{e g}^{*} \cdot \mathbf{G}\left(\mathbf{r}_{i}-\mathbf{r}_{j}, \omega_{e g}\right) \cdot \mathbf{d}_{e g} e^{i \mathbf{k}_{p} \cdot\left(\mathbf{r}_{i}-\mathbf{r}_{j}\right)}$ factor in the first line of Eq. (B7) is carefully evaluated as follows: For $i=j$, we have divergent $\mathbf{d}_{e g}^{*} \cdot \mathbf{G}\left(0^{+}, \omega_{e g}\right) \cdot \mathbf{d}_{e g}$ whose real part accounts for the single-atom Lamb shift and is absorbed into a redefinition of $\omega_{e g}$, with imaginary part equal to $\Gamma / 2$ for isolated two-level atoms. The $i \neq j$ part is evaluated after the $\left\{\mathbf{r}_{i}\right\}$-configuration average as $V^{\prime}=N \frac{k_{p}^{2}\left|\mathbf{d}_{g g}\right|^{2}}{\varepsilon_{0}} \iint \mathbf{d}_{e g}^{*} \cdot \mathbf{G}\left(\mathbf{r}-\mathbf{r}^{\prime}\right) \cdot \mathbf{d}_{e g} \varrho(\mathbf{r}) \varrho\left(\mathbf{r}^{\prime}\right) e^{i \mathbf{k}_{p} \cdot\left(\mathbf{r}-\mathbf{r}^{\prime}\right)} d^{3} \mathbf{r} d^{3} \mathbf{r}^{\prime}$. Following the same integration trick to arrive at Eq. (3), we rewrite this integration into the form of $V^{\prime} \propto \int \overline{\boldsymbol{\varepsilon}_{p}}(\mathbf{r}) \varrho(\mathbf{r})$ to have

$$
\begin{aligned}
V^{\prime} & \approx \frac{N \sigma_{r} \Gamma}{4 i} \int d^{2} r_{\perp} \int d r_{p} \int^{r_{p}} d r_{p}^{\prime} \varrho\left(\mathbf{r}_{\perp}, r_{p}^{\prime}\right) \varrho\left(\mathbf{r}_{\perp}, r_{p}\right), \\
& =-\frac{i}{8} \overline{\mathrm{OD}}_{p} \Gamma
\end{aligned}
$$

with the normalized column density $\varrho_{c}\left(\mathbf{r}_{\perp}\right)=\int \varrho\left(\mathbf{r}_{\perp}, r_{p}\right) d r_{p}$ and with $\overline{\mathrm{OD}}_{p}=N \sigma_{r} \int \varrho_{c}\left(\mathbf{r}_{\perp}\right)^{2} d^{2} r_{\perp}$, as in the main text. We finally have

$$
\overline{V_{\mathrm{DD}}}\left(\mathbf{k}_{p}, \mathbf{k}_{p}\right) \approx-\frac{i}{2}\left(1+\frac{\overline{\mathrm{OD}}_{p}}{4}\right) \Gamma .
$$

To obtain the simple expression of $V^{\prime}$ in Eq. (B8) and $\overline{V_{\mathrm{DD}}}\left(\mathbf{k}_{p}, \mathbf{k}_{p}\right)$ in Eq. (B9), the SVE and Raman-Nath approximations are applied to evaluate $\overline{\boldsymbol{\varepsilon}_{p}}$ inside the sample. The approximations lead to field errors of order $\lambda_{p} / \sigma$ or higher. The corrections of these errors are associated with densitydependent corrections to Eq. (B9), including the collective Lamb shifts [16].

We come back to Eq. (B7). For the smooth density distribution at moderate densities under consideration, the intermode couplings $V_{\mathrm{DD}}\left(n, \mathbf{k}_{p}\right)$ are generally expected to be quite weak and $\left\{\mathbf{r}_{i}\right\}$-specific. For the $\left\{\mathbf{r}_{i}\right\}$-averaged fields, at an observation location $\mathbf{r}_{o}$ in the far field along the $\mathbf{k}_{p}$ direction, the couplings can be completely ignored initially, since with $\mathbf{G}\left(\mathbf{r}_{o}-\mathbf{r}_{i}, \omega_{e g}\right) \propto \frac{e^{i\left(k_{p} r_{o}-\mathbf{k}_{p} \mathbf{r}_{i}\right)}}{4 \pi r_{r_{o}}}$ we have $\overline{\boldsymbol{\varepsilon}_{p}}\left(\mathbf{r}_{o}, 0\right) \propto \int \varrho(\mathbf{r}) d^{3} \mathbf{r}$ while $\overline{\boldsymbol{\varepsilon}_{n}}\left(\mathbf{r}_{o}, 0\right)=0$. We consider $\boldsymbol{\varepsilon}_{p}=\overline{\boldsymbol{\varepsilon}_{p}}+\delta \boldsymbol{\varepsilon}_{p}, \boldsymbol{\varepsilon}_{n}=\overline{\boldsymbol{\varepsilon}_{n}}+$ $\delta \boldsymbol{\varepsilon}_{n}, V_{\mathrm{DD}}=\overline{V_{\mathrm{DD}}}+\delta V_{\mathrm{DD}}$, and apply the $\left\{\mathbf{r}_{i}\right\}$-configuration average to Eq. (B7). By ignoring the $\overline{\varepsilon_{n}}$ terms, we obtain the initial decay of $\boldsymbol{\varepsilon}_{p}\left(\mathbf{r}_{o}, t\right)$ as

$$
\dot{\overline{\boldsymbol{\varepsilon}_{p}}}\left(\mathbf{r}_{o}, t\right) \approx-i \overline{\overline{\mathrm{DD}}}\left(\mathbf{k}_{p}, \mathbf{k}_{p}\right) \overline{\overline{\boldsymbol{\varepsilon}_{p}}}\left(\mathbf{r}_{o}, t\right)+O(\langle\delta V \delta \boldsymbol{\varepsilon}\rangle) .
$$

Equations (B9) and (B10) suggest superradiant decay of directional spontaneous emission power at the $\Gamma_{N}=$ $\left(1+\overline{\mathrm{OD}}_{p} / 4\right) \Gamma$ rate on the exact forward $\left(\mathbf{k}_{p}\right)$ direction for atomic samples at moderate densities $\left(N<k_{p}^{3} \sigma^{3}\right)$. Apart from predicting the decay rate of the far-field emission, it is worth pointing out that the $\Gamma_{N}=2 \operatorname{Im}\left\langle\psi_{\mathrm{TD}}\left(\mathbf{k}_{p}\right)\left|H_{\mathrm{eff}}\right| \psi_{\mathrm{TD}}\left(\mathbf{k}_{p}\right)\right\rangle$ associated with Eq. (B9) is also applicable to the decay of $\left|\psi_{\mathrm{TD}}\left(\mathbf{k}_{p}\right)\right\rangle$ population in the Schrödinger picture $[10,11,13,16,82,84,85]$, and by energy conservation the initial rate of photon emission into $4 \pi$. In this paper, we further approximately identify this decay rate with that for the observable $i_{\mathbf{k}_{p}}(t) \propto \int d^{2} r_{\perp}\left|\overline{\varepsilon_{p}}\left(\mathbf{r}_{\perp}, t\right)\right|^{2}$, leading to Eq. (4) in the main text for the collective emission. The same conclusion can be reached if one simply assumes the spatial profile $\boldsymbol{\varepsilon}_{p}(\mathbf{r}, t)$ would not change significantly during the emission, so as to ignore the $V_{\mathrm{DD}}\left(n, \mathbf{k}_{p}\right)$ couplings. However, it is important to note that for $\boldsymbol{\varepsilon}_{n}$ in Eq. (B7) associated with collective emission near the forward directions (close to $\mathbf{k}_{p}$ ), the $V_{\mathrm{DD}}\left(n, \mathbf{k}_{p}\right)$ couplings can also be collective, and may strongly affect $\boldsymbol{\varepsilon}_{p}(\mathbf{r}, t)$ dynamics at $\mathbf{r}$ along similar directions. Such couplings are just small angle diffractions by the averaged sample profile that generally lead to reshaped emission wavefronts $\boldsymbol{\varepsilon}_{p}(\mathbf{r}, t)$ over time [52], and, as a consequence, deviation of $i_{\mathbf{k}_{p}}(t)$ decay rate from that for the $\left|\psi_{\mathrm{TD}}\left(\mathbf{k}_{p}\right)\right\rangle$ population. The last term in Eq. (B10) is associated with granularity of the atomic distribution, and we also expect that such granularity cannot be ignored for very high densities or for systems with broken symmetry, such as in a lattice.

In future work [49], it would be interesting to better understand the effect of discreteness on collective interactions, and in addition to investigate further the corrections due to the intermode coupling in Eq. (B7) and the possible deviation from the dynamics of Eq. (4).

We remark that in all discussions in this paper, the replacement $\left|\mathbf{d}_{e g}\right|^{2}=\hbar \Gamma \alpha_{i} / 2$ is general and applicable to atoms with level degeneracy. Thus we expect the conclusions for Eqs. (3)-(6) in the main text applicable to the $D 2$ line of ${ }^{87} \mathrm{Rb}$ atom in this paper.

\section{Simulation of spin-wave dynamics supported by noninteracting atoms}

We now ignore the atom-atom interaction in Eq. (B1) and write down the effective non-Hermitian Hamiltonian for the interaction-free model of $N$ three-level atoms as

$$
H_{\mathrm{eff}}^{\prime}=\sum_{i=1}^{N}\left(H_{a}^{i}+H_{e}^{i}-i \hbar \frac{\Gamma_{D 2}}{2}\left|e_{i}\right\rangle\left\langle e_{i}\left|-i \hbar \frac{\Gamma_{D 1}}{2}\right| a_{i}\right\rangle\left\langle a_{i}\right|\right),
$$

where

$$
H_{a}^{i}=-\hbar \Delta\left|a_{i}\right\rangle\left\langle a_{i}\right|+\frac{\hbar}{2}\left(\eta\left(\mathbf{r}_{i}\right) \Omega_{c}(t) e^{-i \varphi_{c}\left(\mathbf{r}_{i}, t\right)} \sigma_{c, i}^{+}+\text {H.c. }\right)
$$

and

$$
H_{e}^{i}=-\hbar \Delta_{e}\left|e_{i}\right\rangle\left\langle e_{i}\right|+\frac{\hbar}{2}\left(\Omega_{p}(t) e^{-i \varphi_{p}\left(\mathbf{r}_{i}\right)} \sigma_{i}^{+}+\text {H.c. }\right) .
$$

Here, $\mathbf{r}_{i}$ is the spatial position of the $i$ th atom and we have $\sigma_{c, i}^{+}=\left|a_{i}\right\rangle\left\langle g_{i}\right|$ and $\sigma_{i}^{+}=\left|e_{i}\right\rangle\left\langle g_{i}\right|$. The control Rabi frequency is $\eta\left(\mathbf{r}_{i}\right) \Omega_{c}(t)=\left|\mathbf{E}_{c}\left(\mathbf{r}_{i}, t\right) \cdot \mathbf{d}_{a g}\right| / \hbar$, with a Gaussian beam intensity profile. $\Omega_{c}(t)$ is the spatially peak value of the control Rabi frequency and $\eta\left(\mathbf{r}_{i}\right) \leqslant 1$ is a position-dependent factor. $\Omega_{p}$ is the Rabi frequency of the probe pulse with $\int_{-\tau_{p}}^{0} \Omega_{p} d t \ll 1$ as defined before. $\varphi_{p}\left(\mathbf{r}_{i}\right)=-\mathbf{k}_{p} \cdot \mathbf{r}_{i}$ is the optical phase of 


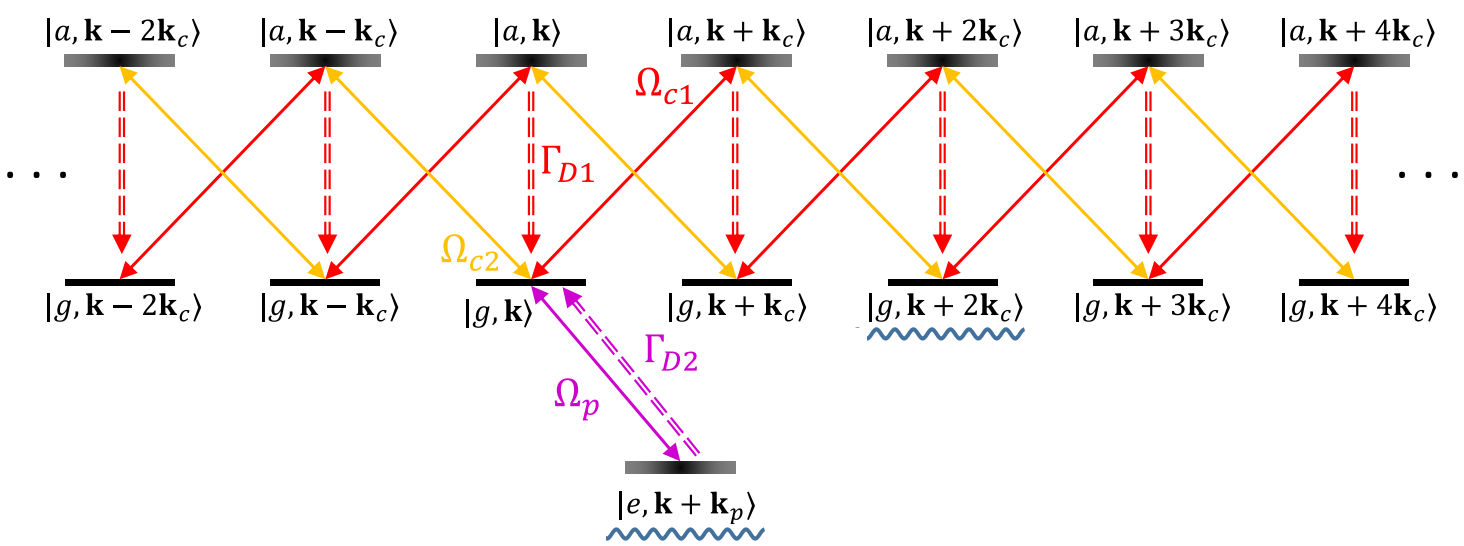

FIG. 7. Momentum lattice structure for probe excitation and $U_{g}$ control simulations according to Eqs. (B14)-(B16). Dash arrows represent the effective quantum jump operations associated with Eq. (B16). The double-sided arrows represent the coherent laser couplings. The coherence between the wavy underlined lattice sites $\left|e, \mathbf{k}+\mathbf{k}_{p}\right\rangle$ and $\left|g, \mathbf{k}+2 \mathbf{k}_{c}\right\rangle$ is associated with the redirected superradiant emission.

the probe pulse. $\Omega_{c}(t)$ and $\dot{\varphi}_{c}\left(\mathbf{r}_{i}, t\right)=\delta_{c}\left(\mathbf{r}_{i}, t\right)$ are depicted in Fig. 1(a) in the main text for robust state-dependent phasepatterning. By changing the basis into $\mathbf{k}$ space, we can rewrite the Hamiltonian in Eq. (B11) as $H_{\mathrm{eff}}=\sum H_{\mathrm{eff}}^{(s)}$ with

$$
\begin{aligned}
H_{\mathrm{eff}}^{(s)}= & H_{p}+H_{c 1}+H_{c 2}, \\
H_{p}= & \hbar \sum_{g, \mathbf{k}} \Delta_{g}|g, \mathbf{k}\rangle\langle g, \mathbf{k}| \\
& +\hbar \sum_{e, \mathbf{k}}\left(-\Delta_{e}-i \Gamma_{D 2} / 2\right)|e, \mathbf{k}\rangle\langle e, \mathbf{k}| \\
& +\hbar \sum_{a, \mathbf{k}}\left(-\Delta_{a}-i \Gamma_{D 1} / 2\right)|a, \mathbf{k}\rangle\langle a, \mathbf{k}| \\
& +\hbar \sum_{g, e, \mathbf{k}}\left(\frac{1}{2} \Omega_{p}\left(t+\tau_{p}\right) c_{e g}^{y}\left|e, \mathbf{k}+\mathbf{k}_{p}\right\rangle\langle g, \mathbf{k}|+\text { H.c. }\right), \\
H_{c 1}= & -\hbar \sum_{a, \mathbf{k}} \delta_{c}\left(t-t_{1}\right)|a, \mathbf{k}\rangle\langle a, \mathbf{k}| \\
& +\hbar \sum_{g, a, \mathbf{k}}\left(\frac{1}{2} \eta_{1} \Omega_{c 1}\left(t-t_{1}\right) c_{a g}^{x}\left|a, \mathbf{k}+\mathbf{k}_{c}\right\rangle\langle g, \mathbf{k}|+\text { H.c. }\right), \\
& -\hbar \sum_{a, \mathbf{k}} \delta_{c}\left(t-t_{2}\right)|a, \mathbf{k}\rangle\langle a, \mathbf{k}| \\
H_{c 2}= & \hbar \sum_{g, a, \mathbf{k}}\left(\frac{1}{2} \eta_{2} \Omega_{c 2}\left(t-t_{2}\right) c_{a g}^{x}\left|a, \mathbf{k}-\mathbf{k}_{c}\right\rangle\langle g, \mathbf{k}|+\text { H.c. }\right), \\
& +\hbar 14)
\end{aligned}
$$

where $|g, \mathbf{k}\rangle=\frac{1}{\sqrt{N}} \sum_{i} e^{i \mathbf{k} \cdot \mathbf{r}_{i}}\left|g_{i}\right\rangle$ and similarly for $|e, \mathbf{k}\rangle$ and $|a, \mathbf{k}\rangle$. Here we have included all the $D 1$ and $D 2$ hyperfine levels and use $\{g, e, a\}$ as indices to label the $\left\{5 S_{1 / 2}, 5 P_{3 / 2}, 5 P_{1 / 2}\right\}$ hyperfine levels, respectively. The $c_{a g}^{x}, c_{e g}^{y}$ are coupling coefficients for $\mathbf{e}_{x}-$ and $\mathbf{e}_{y}-$ polarized $D 1$ and $D 2$ pulses, derived from the Clebsch-Gorden coefficients. Here $\eta_{1,2}$ are factors to account for the laser intensity inhomogeneities. Following the convention as in Fig. 1(a), the probe excitation is between $-\tau_{p}<t<0$, which is followed by the two $D 1$ control pulses $\left(H_{c 1}\right.$ and $\left.H_{c 2}\right)$ starting at $t_{1}=\Delta t_{1}$ and $t_{2}=\Delta t_{1}+\tau_{d}$, respectively. We finally end up with the master equation for the single-atom density matrix $\rho^{(s)}$ as

$$
\begin{aligned}
\dot{\rho}^{(s)}(t)= & \frac{1}{i}\left(H_{\mathrm{eff}}^{(s)} \rho^{(s)}-\rho^{(s)} H_{\mathrm{eff}}^{(s) \dagger}\right) \\
& +\sum_{j}\left(\hat{C}_{D 1}^{j} \rho^{(s)} \hat{C}_{D 1}^{j \dagger}+\hat{C}_{D 2}^{j} \rho^{(s)} \hat{C}_{D 2}^{j \dagger}\right) .
\end{aligned}
$$

The collapse operators are simply defined as

$$
\begin{aligned}
& \hat{C}_{D 1}^{j}=\sum_{a, g, \mathbf{k}} \sqrt{\Gamma_{D 1}} c_{a g}^{j}|g, \mathbf{k}\rangle\langle a, \mathbf{k}|, \\
& \hat{C}_{D 2}^{j}=\sum_{e, g, \mathbf{k}} \sqrt{\Gamma_{D 2}} c_{e g}^{j}|g, \mathbf{k}\rangle\left\langle e, \mathbf{k}+\mathbf{k}_{p}\right|,
\end{aligned}
$$

with $j$ running through $x, y$, and $z$ polarizations. This effective evolution of the density matrix within the momentum lattice basis is summarized in Fig. 7.

With $\rho^{(s)}(t)$ it is straightforward to calculate the interaction-free evolution of the many-atom density matrix $\rho(t)=\left(\rho^{(s)}(t)\right)^{\otimes N}$ and to evaluate collective observables $\langle\hat{O}\rangle=\operatorname{tr}(\rho(t) \hat{O})$ [30]. By solving the master equation with the initial condition $\rho^{(s)}=\frac{1}{5} \sum_{g=1}^{5}|g, \mathbf{k}\rangle\langle g, \mathbf{k}|$ (with $|g\rangle$ running through the $\left|F=2, m_{F}\right\rangle$ Zeeman sublevels), we further calculate the dipole coherence $\mathbf{d}\left(\mathbf{k}_{s}\right)=\operatorname{tr}\left(\rho^{(s)}(t) \mathbf{d}^{-}\left(\mathbf{k}_{s}\right)\right)$, and similarly for $\mathbf{d}\left(\mathbf{k}_{p}\right)$ and $\mathbf{d}\left(\mathbf{k}_{s}^{\prime}\right)$. Here we define the operators $\mathbf{d}^{-}\left(\mathbf{k}_{s}\right)=\mathbf{e}_{y} \sum_{g, e} c_{e g}^{y}\left|g, \mathbf{k}+2 \mathbf{k}_{c}\right\rangle\left\langle e, \mathbf{k}+\mathbf{k}_{p}\right|, \mathbf{d}^{-}\left(\mathbf{k}_{p}\right)=$ $\mathbf{e}_{y} \sum_{g, e} c_{e g}^{y}|g, \mathbf{k}\rangle\left\langle e, \mathbf{k}+\mathbf{k}_{p}\right|$ and $\mathbf{d}^{-}\left(\mathbf{k}_{s}^{\prime}\right)=\mathbf{e}_{y} \sum_{g, e} c_{e g}^{y} \mid g, \mathbf{k}+$ $\left.4 \mathbf{k}_{c}\right\rangle\left\langle e, \mathbf{k}+\mathbf{k}_{p}\right|$. The superradiant signal $i_{\mathbf{k}_{s}}$ in the main text is related to the expectation value $\left\langle S^{+}\left(\mathbf{k}_{s}\right) S^{-}\left(\mathbf{k}_{s}\right)\right\rangle$. In the large $N$ limit, we approximately have $\left\langle S^{+}\left(\mathbf{k}_{s}\right) S^{-}\left(\mathbf{k}_{s}\right)\right\rangle \approx$ $\left|\left\langle S^{-}\left(\mathbf{k}_{s}\right)\right\rangle\right|^{2}$, where $\left\langle S^{-}\left(\mathbf{k}_{s}\right)\right\rangle$ is proportional to the dipole coherence $\left\langle\mathbf{d}^{-}\left(\mathbf{k}_{s}\right)\right\rangle$ as $\left\langle S^{-}\left(\mathbf{k}_{s}\right)\right\rangle \propto N\left\langle\mathbf{d}^{-}\left(\mathbf{k}_{s}\right)\right\rangle$. Thus, by calculating the dipole coherence, the simulation can reproduce the $D 2$ collective emission dynamics with $D 1$ control for noninteracting atoms. With experimental imperfections encoded in parameters like $\eta_{1,2}$ in Eq. (B14), we refer to the numerically evaluated single-atom density matrix according to Eq. (B15) as $\rho_{\eta}^{(s)}(t)$. For comparison, the perfect geometric phase patterning is implemented by replacing the evolution by $H_{c 1}+H_{c 2}$ in Eq. (B14) with instantaneous $U_{g}\left(-2 \mathbf{k}_{c}\right)=1-$ $\sum_{g}|g, \mathbf{k}\rangle\left\langle g, \mathbf{k}\left|+\sum_{g}\right| g, \mathbf{k}+2 \mathbf{k}_{c}\right\rangle\langle g, \mathbf{k}|$, leading to a perfectly 
controlled density matrix $\rho_{0}^{(s)}(t)$ for $t>0$. We then further define $i_{s, \eta}(t)=\left|\operatorname{tr}\left(\rho_{\eta}^{(s)}(t) \mathbf{d}\left(\mathbf{k}_{s}\right)\right)\right|^{2}$ and similarly $i_{s, 0}(t)=$ $\left|\operatorname{tr}\left(\rho_{0}^{(s)}(t) \mathbf{d}\left(\mathbf{k}_{s}\right)\right)\right|^{2}$ for redirected superradiance under perfect control.

\section{3. $f_{d}$ and $f_{a}$ estimation}

We relate the experimental observable $i_{\mathbf{k}_{s}}(t)$ with ensemble-averaged $i_{s, \eta}(t)$ as $\left\langle i_{s, \eta}\right\rangle_{\eta}$, and calculate collective dipole control efficiency as $f_{d}=\left\langle i_{s, \eta}\left(\tau_{c}+\tau_{d}\right)\right\rangle_{\eta} / i_{s, 0}(0)$. The ensemble average of emission intensity, instead of field amplitude, is in light of the fact that we experimentally collect $i_{\mathbf{k}_{s}}(t)$ with a multimode fiber, and the signal $i_{\mathbf{k}_{s}}(t)$ is insensitive to slight distortion of the $\mathbf{E}_{s}$-mode profile by the dynamic phase writing due to the imbalanced $\eta_{1,2}$.

The simulation of optical acceleration by the $D 1$ control pulses follows the same Eqs. (B14) and (B15) but without the probe excitation and with atomic levels restricted to the $D 1$ line only. We evaluate the momentum transfer as $\Delta P_{\eta}=\hbar \mathbf{k}_{c}\left(\sum_{g, n} n\left\langle g, \mathbf{k}+n \mathbf{k}_{c}\left|\rho_{\eta}^{(s)}(t)\right| g, \mathbf{k}+\right.\right.$ $\left.\left.n \mathbf{k}_{c}\right\rangle+\sum_{a, n} n\left\langle a, \mathbf{k}+n \mathbf{k}_{c}\left|\rho_{\eta}^{(s)}(t)\right| a, \mathbf{k}+n \mathbf{k}_{c}\right\rangle\right)$ for $t=\tau_{c}+$ $\tau_{d}$. We then compare the ensemble-averaged acceleration efficiency $f_{a}=\left\langle\Delta P_{\eta}\right\rangle_{\eta} /\left(2 \hbar k_{c}\right)$ with the experimental measurements.

The $\eta_{1,2}$ average in both calculations is according to spatial distribution of the control laser beam intensity profile seen by the atomic sample. As the final results are quite insensitive to distribution details, we assume both the laser beam and the atomic sample have Gaussian profiles, with waists $w=$ $13 \mu \mathrm{m}$ and $\sigma=7 \mu \mathrm{m}$ by fitting the imaging measurements and with optics simulations. We adjust the retroreflected beam waist $w_{r}$ and the intensity factor $\eta_{2} \propto 1 / w_{r}$ accordingly in the simulation, together with an overall intensity calibration factor $\kappa$ multiplied to the $s$ parameter from the beat-note measurements of the control pulses [30]. The ensemble-averaged $f_{a}$ is compared with experimentally measured $\Delta P / 2 \hbar k_{c}$ and we adjust $\kappa, w_{r}$ to globally match the single-atom simulation with all the measurement results for optical acceleration as in Fig. 6. We then estimate both $f_{a}, f_{d}$ as discussed in Sec. III E. Since the second type of delay line [Fig. 2(b)] involves more optics than the first one [Fig. 2(a)], we expect more power loss and wavefront distortion for the retroreflected pulses. However, with the second-type delay line, we are able to experimentally adjust the amplitude of the preprogrammed pulses [marked with " $p$ " in Fig. 2(b)] to approximately rebalance the intensity of the counterpropagating control pulses despite the power loss. In the corresponding simulation, we accordingly readjust the beam waist $w_{r}$ for the retroreflection together with the intensity factor $\eta_{2} \propto \alpha / w_{r}$. Within reasonable adjustments, the simulation results (see Fig. 8) suggest that the efficiency $f_{d}$ of the single $\mathbf{k}$-shift operation reaches $75 \%$, agreeing with the estimation based on the retrieval efficiency [30].

\section{Reconstructing the controlled spin-wave dynamics}

With the simulation parameters optimally matching the experiment, we further simulate the experimental sequence in Fig. 4 and calculate $\left\langle|\mathbf{d}(\mathbf{k})|^{2}\right\rangle=\left\langle\left|\operatorname{tr}\left(\rho_{\eta}^{(s)}(t) \mathbf{d}(\mathbf{k})\right)\right|^{2}\right\rangle_{\eta}$ associated with collective dipole excitation with $\mathbf{k}=\left\{\mathbf{k}_{p}, \mathbf{k}_{s}=\right.$ $\left.\mathbf{k}_{p}-2 \mathbf{k}_{c}, \mathbf{k}_{s}^{\prime}=\mathbf{k}_{p}-4 \mathbf{k}_{c}\right\}$ for the forward, redirected, and

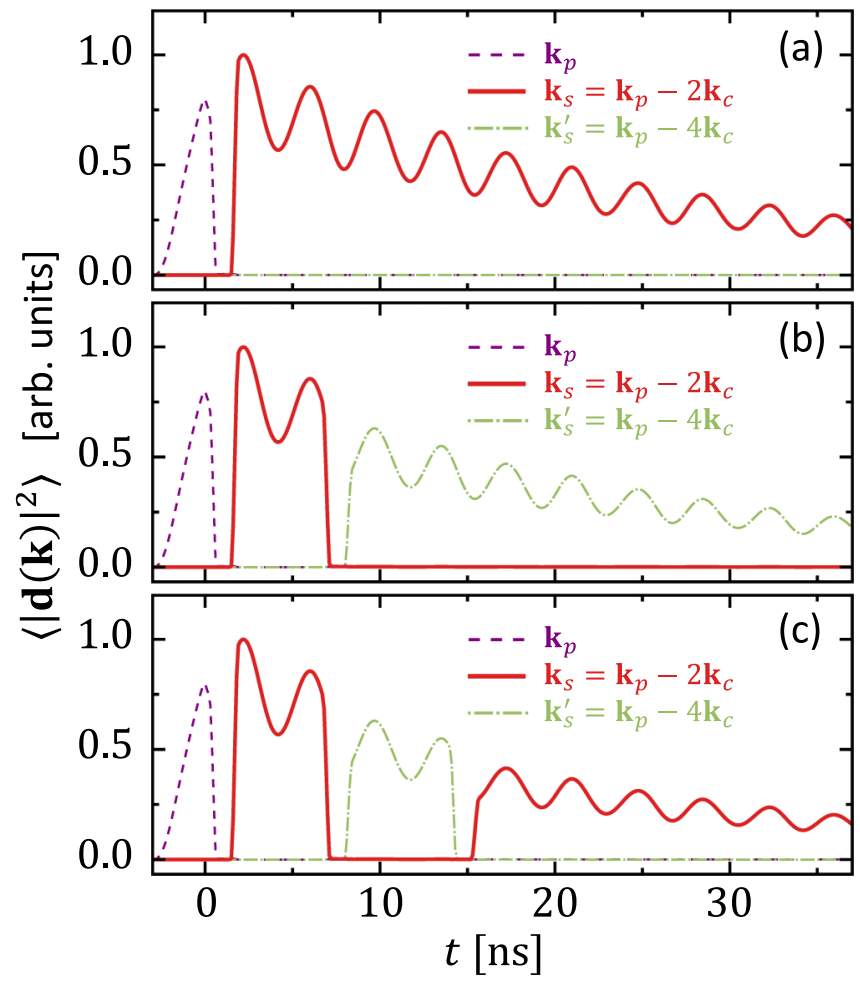

FIG. 8. Simulation of spin-wave dynamics for typical experimental sequences. The spin-wave intensities represented by $\left\langle|\mathbf{d}(\mathbf{k}, t)|^{2}\right\rangle$ are evaluated according to Eq. (B15) with optimally estimated experimental parameters. (a) is according to Eq. (B14) with a $\tau_{p}=3 \mathrm{~ns} D 2$ probe excitation followed by a $\mathbf{k} \rightarrow \mathbf{k}-2 \mathbf{k}_{c}$ control composed of two chirped $D 1$ pulses with $\tau_{c}=0.5 \mathrm{~ns}$ and $\tau_{d}=1.24$ ns at $\Delta t_{1}=0.2 \mathrm{~ns}$. The $\mathbf{k}_{p}$ (corresponds to forward radiation) and $\mathbf{k}_{s}=\mathbf{k}_{p}-2 \mathbf{k}_{c}$ (corresponds to the redirected radiation) spin-wave components are plotted with dashed and solid lines, respectively. The dash-dotted line corresponds to the mismatched $\mathbf{k}_{s}^{\prime}=\mathbf{k}_{p}-4 \mathbf{k}_{c}$ excitation. In (b), an additional $\mathbf{k} \rightarrow \mathbf{k}-2 \mathbf{k}_{c}$ is applied with $\Delta t_{2}=$ $4.8 \mathrm{~ns}$. In (c), a $\mathbf{k} \rightarrow \mathbf{k}+2 \mathbf{k}_{c}$ recall is simulated at $\Delta t_{3}=5.5 \mathrm{~ns}$. A recall efficiency similar to those in Fig. 4 is recovered. The $\sim 267 \mathrm{MHz}$ quantum beat amplitude needs to be taken into account when comparing the spin-wave amplitudes and to estimate the control efficiency. Notice the difference of $\tau_{p}$ and thus a different contrast to the quantum beat comparing with measurements in Fig. 4.

subradiantly stored collective radiation, respectively. We not only reproduce features of the experimental observable $i_{\mathbf{k}_{s}}(t) \propto\left\langle\left|\mathbf{d}\left(\mathbf{k}_{s}\right)\right|^{2}\right\rangle$ but also unveil time-dependent dynamics for the unmonitored forward emission $\left\langle\left|\mathbf{d}\left(\mathbf{k}_{p}\right)\right|^{2}\right\rangle$ and the subradiantly stored or the superradiance-free excitation $\left\langle\left|\mathbf{d}\left(\mathbf{k}_{s}^{\prime}\right)\right|^{2}\right\rangle$. Typical results are given in Fig. 8.

\section{Consistent recall at selected $\Delta t_{3}$ delay}

The redirection-switch off-recall sequence as in Fig. 4 involves multiple spin-wave controls $U_{g}\left( \pm 2 \mathbf{k}_{c}\right)$ implemented with cyclic $D 1$ transition, driven by pairs of counterpropagating control pulses. The controls are not perfect. In particular, after each pair of pulsed control, there is residual $D 1$ population left in the $5 P_{1 / 2}$ state with dipole coherence determined by the optical phases as well as details of the control dynamics. For two successive pulses of the same type, the coherently 

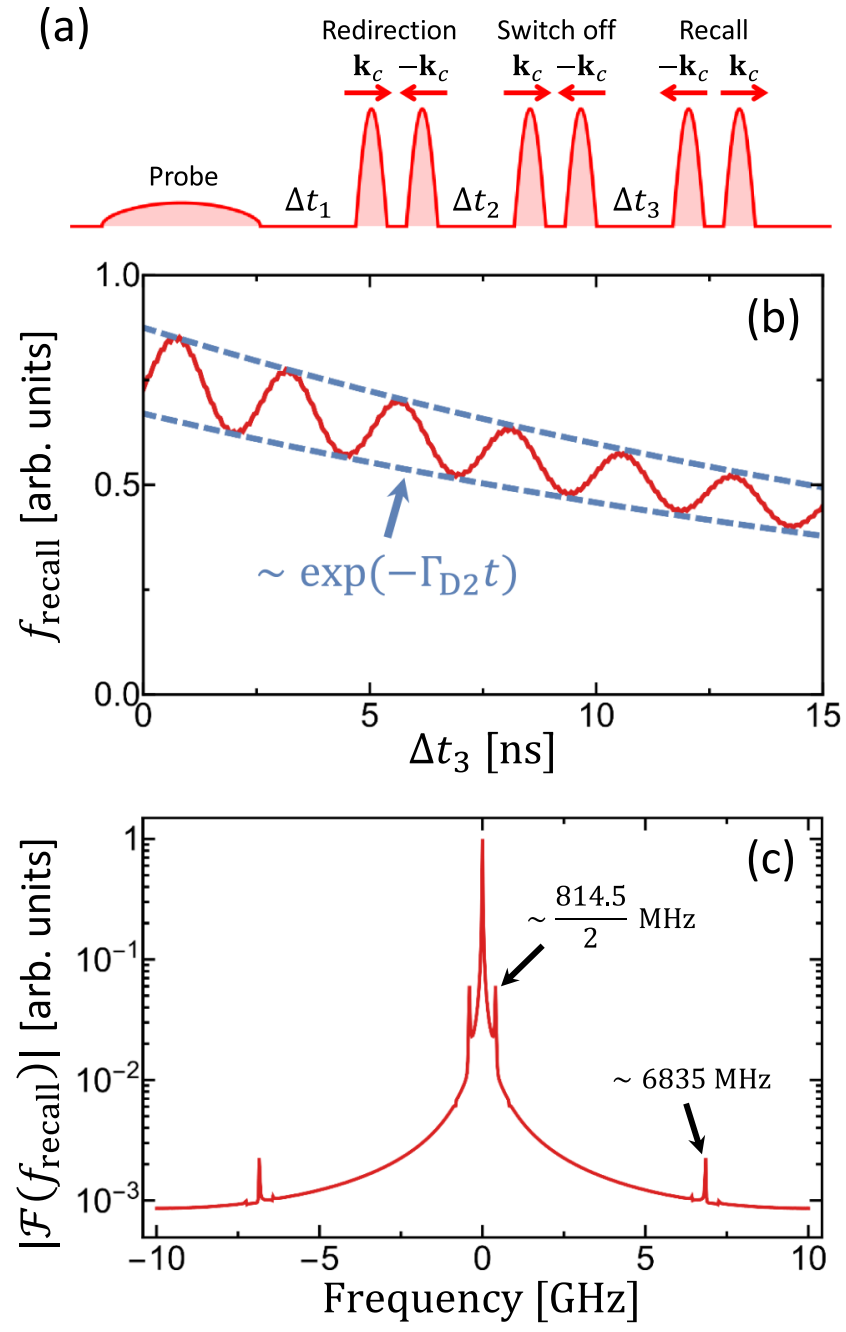

FIG. 9. Simulation of residual $D 1$ dipole coherence induced interference effect for the recall with various $\Delta t_{3}$. (a) The pulse sequence of the simulation, according to the experiments. (b) We scan $\Delta t_{3}$ with all the other parameters in the full redirection-switch off-recall sequence unchanged and numerically calculate the relative recall efficiency. Blue dashed line: Reference of single decay dynamics. (c) The Fourier transform of the $\Delta t_{3}$-dependent recall efficiency. added residual $D 1$ coherences may be enhanced or canceled, depending on the relative phase between the two. Such interference effects are actually explored for robust quantum control with composite pulse techniques $[62,86]$.

In this experiment, the interference effect emerges, in particular, between the second pulse of the switch-off control and the first pulse of the recall control, both driven by a $-\mathbf{k}_{c}$ chirped pulse [Fig. 9(a)]. The relative phase between the dipole coherence evolves according to the laser detuning to the dipole transitions. Here, with the center frequency of the control pulse at the midpoint of the hyperfine transitions $5 S_{1 / 2} F=2-5 P_{1 / 2} F^{\prime}=1,2$, the interference leads to oscillation of the control efficiency, which is expected at the frequency $\Delta_{D 1, \mathrm{hfse}} / 4 \pi=814.5 / 2 \mathrm{MHz}\left(\Delta_{D 1 \text {,hfse }}\right.$ is the $5 P_{1 / 2}$ hyperfine splitting.). The oscillations have been observed experimentally. To confirm the picture, we did a single-body simulation including all hyperfine levels in the $D 1$ and $D 2$ line of ${ }^{87} \mathrm{Rb}$ as modeled in Appendix B 2, by setting the Hamiltonian $H_{c 1}+H_{c 2}$ to match the sequence in the Fig. 1(a) of the main text and furthermore with control parameters consistent with the experimental condition. All the parameters are fixed except the recall delay $\Delta t_{3}$, which is scanned in simulations to numerically evaluate a relative recall efficiency define as $\left.f_{\text {recall }} \propto\left(\left|\mathbf{d}\left(\mathbf{k}_{s}\right)\right|^{2}\right)\right|_{t=3 \tau_{c}+3 \tau_{d}+\Delta t_{1}+\Delta t_{2}+\Delta t_{3}}$. The simulation results are shown in Fig. 9. As expected, the recall efficiency oscillates with $\Delta t_{3}$ with $\Delta_{D 1 \text {,hfse }} / 2$ frequency. In addition, it should be noted that there is oscillation at the frequency $\sim 6.8 \mathrm{GHz}$ with much smaller amplitude [Fig. 9(b)], which is associated with the hyperfine splitting of the ground state. This additional modulation of the recall efficiency is because the dipole coherence in the hyperfine transitions $5 S_{1 / 2} F=1-5 P_{1 / 2} F^{\prime}=1,2$ is also excited during the control, though inefficiently since the control pulse is far-detuned and negligibly influences our measurements.

To make sure that the residual dipole coherence interference effect is consistent for all recall delays, we carefully select the recall delay time to be $\Delta t_{3}=4 \pi m / \Delta_{D 1 \text {,hfse }}+t_{\text {off }}$, with integers $m$ and a constant offset $t_{\text {off }}$. This recall time selection makes it possible for us to study the decay dynamics of the phase-mismatched spin waves with a suppressed systematic error induced by the imperfect $D 1$ control.
[1] C. H. Bennett and D. P. Divincenzo, Quantum information and computation, Nature (London) 404, 247 (2000).

[2] J. Dalibard, F. Gerbier, G. Juzeliūnas, and P. Öhberg, Colloquium: Artificial gauge potentials for neutral atoms, Rev. Mod. Phys. 83, 1523 (2011).

[3] I. M. Georgescu, S. Ashhab, and F. Nori, Quantum simulation, Rev. Mod. Phys. 86, 153 (2014).

[4] C. L. Degen, F. Reinhard, and P. Cappellaro, Quantum sensing, Rev. Mod. Phys. 89, 035002 (2017).

[5] K. Hammerer, A. S. Sørensen, and E. S. Polzik, Quantum interface between light and atomic ensembles, Rev. Mod. Phys. 82, 1041 (2010).

[6] K. Heshami, D. G. England, P. C. Humphreys, P. J. Bustard, V. M. Acosta, J. Nunn, and B. J. Sussman, Quantum memories:
Emerging applications and recent advances, J. Mod. Opt. 63, 2005 (2016).

[7] R. H. Dicke, Coherence in spontaneous radiation processes, Phys. Rev. 93, 99 (1954).

[8] S. Zhang, C. Liu, S. Zhou, C.-S. Chuu, M. M. T. Loy, and S. Du, Coherent Control of Single-Photon Absorption and Reemission in a Two-Level Atomic Ensemble, Phys. Rev. Lett. 109, 263601 (2012).

[9] M. O. Scully, E. S. Fry, C. H. R. Ooi, and K. Wódkiewicz, Directed Spontaneous Emission from an Extended Ensemble of $N$ Atoms: Timing is Everything, Phys. Rev. Lett. 96, 010501 (2006).

[10] M. O. Araújo, I. Krešić, R. Kaiser, and W. Guerin, Superradiance in a Large and Dilute Cloud of Cold Atoms 
in the Linear-Optics Regime, Phys. Rev. Lett. 117, 073002 (2016).

[11] S. J. Roof, K. J. Kemp, M. D. Havey, and I. M. Sokolov, Observation of Single-Photon Superradiance and the Cooperative Lamb Shift in an Extended Sample of Cold Atoms, Phys. Rev. Lett. 117, 073003 (2016).

[12] M. Fleischhauer, A. Imamoglu, and J. P. Marangos, Electromagnetically induced transparency: Optics in coherent media, Rev. Mod. Phys. 77, 633 (2005)

[13] B. Zhu, J. Cooper, J. Ye, and A. M. Rey, Light scattering from dense cold atomic media, Phys. Rev. A 94, 023612 (2016).

[14] F. Andreoli, M. J. Gullans, A. A. High, A. Browaeys, and D. E. Chang, The maximum refractive index of an atomic medium, arXiv:2006.01680.

[15] S. L. Bromley, B. Zhu, M. Bishof, X. Zhang, T. Bothwell, J. Schachenmayer, T. L. Nicholson, R. Kaiser, S. F. Yelin, M. D. Lukin, A. M. Rey, and J. Ye, Collective atomic scattering and motional effects in a dense coherent medium, Nat. Commun. 7, 11039 (2016).

[16] M. O. Scully, Collective Lamb Shift in Single Photon Dicke Superradiance, Phys. Rev. Lett. 102, 143601 (2009).

[17] W. Guerin, M. T. Rouabah, and R. Kaiser, Light interacting with atomic ensembles: Collective, cooperative and mesoscopic effects, J. Mod. Opt. 64, 895 (2017).

[18] S.-T. Chui, S. Du, and G.-B. Jo, Subwavelength transportation of light with atomic resonances, Phys. Rev. A 92, 053826 (2015).

[19] R. J. Bettles, S. A. Gardiner, and C. S. Adams, Cooperative eigenmodes and scattering in one-dimensional atomic arrays, Phys. Rev. A 94, 043844 (2016).

[20] J. A. Needham, I. Lesanovsky, and B. Olmos, Subradianceprotected excitation transport, New J. Phys. 21, 073061 (2019).

[21] R. J. Bettles, S. A. Gardiner, and C. S. Adams, Enhanced Optical Cross Section via Collective Coupling of Atomic Dipoles in a 2D Array, Phys. Rev. Lett. 116, 103602 (2016).

[22] E. Shahmoon, D. S. Wild, M. D. Lukin, and S. F. Yelin, Cooperative Resonances in Light Scattering from Two-Dimensional Atomic Arrays, Phys. Rev. Lett. 118, 113601 (2017).

[23] J. Rui, D. Wei, A. Rubio-Abadal, S. Hollerith, J. Zeiher, D. M. Stamper-Kurn, C. Gross, and I. Bloch, A subradiant optical mirror formed by a single structured atomic layer, Nature (London) $\mathbf{5 8 3}, 369$ (2020).

[24] A. Asenjo-Garcia, M. Moreno-Cardoner, A. Albrecht, H. J. Kimble, and D. E. Chang, Exponential Improvement in Photon Storage Fidelities Using Subradiance and "Selective Radiance" in Atomic Arrays, Phys. Rev. X 7, 031024 (2017).

[25] R. T. Sutherland and F. Robicheaux, Collective dipole-dipole interactions in an atomic array, Phys. Rev. A 94, 013847 (2016).

[26] S. V. Syzranov, M. L. Wall, B. Zhu, V. Gurarie, and A. M. Rey, Emergent Weyl excitations in systems of polar particles, Nat. Commun. 7, 13543 (2016).

[27] J. Perczel, J. Borregaard, D. E. Chang, H. Pichler, S. F. Yelin, P. Zoller, and M. D. Lukin, Topological Quantum Optics in Two-Dimensional Atomic Arrays, Phys. Rev. Lett. 119, 023603 (2017).

[28] R. J. Bettles, J. Mináŕ, C. S. Adams, I. Lesanovsky, and B. Olmos, Topological properties of a dense atomic lattice gas, Phys. Rev. A 96, 041603(R) (2017).
[29] Y.-X. Zhang and K. Mølmer, Theory of Subradiant States of a One-Dimensional Two-Level Atom Chain, Phys. Rev. Lett. 122, 203605 (2019).

[30] Y. He, L. Ji, Y. Wang, L. Qiu, J. Zhao, Y. Ma, X. Huang, S. Wu, and D. E. Chang, Geometric Control of Collective Spontaneous Emission, Phys. Rev. Lett. 125, 213602 (2020).

[31] X. Miao, E. Wertz, M. G. Cohen, and H. Metcalf, Strong optical forces from adiabatic rapid passage, Phys. Rev. A $\mathbf{7 5}$, 011402(R) (2007).

[32] T. Lu, X. Miao, and H. Metcalf, Nonadiabatic transitions in finite-time adiabatic rapid passage, Phys. Rev. A 75, 063422 (2007).

[33] H. Metcalf, Colloquium: Strong optical forces on atoms in multifrequency light, Rev. Mod. Phys. 89, 041001 (2017).

[34] A. M. Jayich, A. C. Vutha, M. T. Hummon, J. V. Porto, and W. C. Campbell, Continuous all-optical deceleration and singlephoton cooling of molecular beams, Phys. Rev. A 89, 023425 (2014).

[35] X. Long, S. S. Yu, A. M. Jayich, and W. C. Campbell, Suppressed Spontaneous Emission for Coherent Momentum Transfer, Phys. Rev. Lett. 123, 033603 (2019).

[36] D.-W. Wang and M. O. Scully, Heisenberg Limit Superradiant Superresolving Metrology, Phys. Rev. Lett. 113, 083601 (2014).

[37] M. O. Scully, Single Photon Subradiance: Quantum Control of Spontaneous Emission and Ultrafast Readout, Phys. Rev. Lett. 115, 243602 (2015).

[38] J. Mizrahi, B. Neyenhuis, K. G. Johnson, W. C. Campbell, C. Senko, D. Hayes, and C. Monroe, Quantum control of qubits and atomic motion using ultrafast laser pulses, Appl. Phys. B 114, 45 (2014).

[39] J. D. Wong-Campos, S. A. Moses, K. G. Johnson, and C. Monroe, Demonstration of Two-Atom Entanglement with Ultrafast Optical Pulses, Phys. Rev. Lett. 119, 230501 (2017).

[40] M. Jaffe, V. Xu, P. Haslinger, H. Müller, and P. Hamilton, Efficient Adiabatic Spin-Dependent Kicks in an Atom Interferometer, Phys. Rev. Lett. 121, 040402 (2018).

[41] S. T. Cundiff and S. Mukamel, Optical multidimensional coherent spectroscopy, Phys. Today 66(7), 44 (2013).

[42] F. D. Fuller and J. P. Ogilvie, Experimental implementations of two-dimensional Fourier transform electronic spectroscopy, Annu. Rev. Phys. Chem. 66, 667 (2015).

[43] T. A. A. Oliver, Recent advances in multidimensional ultrafast spectroscopy, R. Soc. Open Sci. 5, 171425 (2018).

[44] T. Ichikawa, M. Bando, Y. Kondo, and M. Nakahara, Geometric aspects of composite pulses, Phil. Trans. R. Soc. A 370, 4671 (2012).

[45] M. M. T. Loy, Observation of Population Inversion by Optical Adiabatic Rapid Passage, Phys. Rev. Lett. 32, 814 (1974).

[46] S. Zhdanovich, E. A. Shapiro, M. Shapiro, J. W. Hepburn, and V. Milner, Population Transfer Between Two Quantum States by Piecewise Chirping of Femtosecond Pulses: Theory and Experiment, Phys. Rev. Lett. 100, 103004 (2008).

[47] D. Goswami, Optical pulse shaping approaches to coherent control, Phys. Rep. 374, 385 (2003).

[48] M. O. Scully and A. A. Svidzinsky, The super of superradiance, Science 325, 1510 (2009).

[49] The spin-wave control technique combined with modified trap geometries allows us to precisely characterize the phase(mis)matched dipole spin waves dynamics as a function of 
atomic density and number. A paper to present the findings is under preparation.

[50] L. Henriet, J. S. Douglas, D. E. Chang, and A. Albrecht, Critical open-system dynamics in a one-dimensional optical-lattice clock, Phys. Rev. A 99, 023802 (2019).

[51] D. A. Steck, Rubidium 87 D Line Data, http://steck.us/ alkalidata (revision 2.2.1, November 21, 2019).

[52] F. Cottier, R. Kaiser, and R. Bachelard, Role of disorder in super- and subradiance of cold atomic clouds, Phys. Rev. A 98, 013622 (2018).

[53] The data in Fig. 3(b) is from automated measurements over 12 hours with three rounds of automation, with probe power being increased by up to a factor of 5 to enhance the signals for measurements with smallest atom numbers. The adjustments are confirmed not to affect the decay dynamics, and the probe intensities are all well within the linear excitation regime. We recorded the probe light power for each automation. The $i_{\max , N}$ in Fig. 3(b) are relatively normalized by the recorded probe power ratio for the three sets of automation.

[54] M. Cheneau, S. P. Rath, T. Yefsah, K. J. Günter, G. Juzeliūnas, and J. Dalibard, Geometric potentials in quantum optics: A semi-classical interpretation, EPL 83, 60001 (2008).

[55] V. Gritsev and A. Polkovnikov, Dynamical quantum Hall effect in the parameter space, Proc. Natl. Acad. Sci. USA 109, 6457 (2012).

[56] For few-atoms system with long-lived excitations, techniques are developed for frequency-selective excitation of the collective modes, for example, Z. Meir, O. Schwartz, E. Shahmoon, D. Oron, and R. Ozeri, Cooperative Lamb Shift in a Mesoscopic Atomic Array, Phys. Rev. Lett. 113, 193002 (2014); B. H. McGuyer, M. McDonald, G. Z. Iwata, M. G. Tarallo, W. Skomorowski, R. Moszynski, and T. Zelevinsky, Precise study of asymptotic physics with subradiant ultracold molecules, Nat. Phys. 11, 32 (2015); Time-domain control techniques are also developed to manipulate the collective modes, for example, S. de Léséleuc, D. Barredo, V. Lienhard, A. Browaeys, and T. Lahaye, Optical Control of the Resonant Dipole-Dipole Interaction between Rydberg Atoms, Phys. Rev. Lett. 119, 053202 (2017).

[57] As the collective states of many-atom system are typically with unresolved energy differences, precise control of isolated superradiant states in the frequency domain is generally difficult to achieve. However, dynamics of the collective states controlled by continuous-wave lasers with constant lattice couplings is an interesting and emergent topic that has intriguing consequences. For example, superradiance lattices consisting of collective states have been discussed by D.-W. Wang, R.-B. Liu, S.-Y. Zhu, and M. O. Scully, Superradiance Lattice, Phys. Rev. Lett. 114, 043602 (2015); experimentally studied by L. Chen, P. Wang, Z. Meng, L. Huang, H. Cai, D.-W. Wang, S.-Y. Zhu, and J. Zhang, Experimental Observation of OneDimensional Superradiance Lattices in Ultracold Atoms, ibid. 120, 193601 (2018); H. Cai, J. Liu, J. Wu, Y. He, S.-Y. Zhu, J.-X. Zhang, and D.-W. Wang, Experimental Observation of Momentum-Space Chiral Edge Currents in Room-Temperature Atoms, ibid. 122, 023601 (2019), in both cold and hot atomic systems.

[58] Beyond atomic physics, techniques are developed for superand subradiant states conversion in other fields, for example
Z. Wang, H. Li, W. Feng, X. Song, C. Song, W. Liu, Q. Guo, X. Zhang, H. Dong, D. Zheng, H. Wang, and D.-W. Wang, Controllable Switching Between Superradiant and Subradiant States in a 10-Qubit Superconducting Circuit, Phys. Rev. Lett. 124, 013601 (2020).

[59] X. Chen, A. Ruschhaupt, S. Schmidt, A. del Campo, D. Guéry-Odelin, and J. G. Muga, Fast Optimal Frictionless Atom Cooling in Harmonic Traps: Shortcut to Adiabaticity, Phys. Rev. Lett. 104, 063002 (2010).

[60] A. Dunning, R. Gregory, J. Bateman, N. Cooper, M. Himsworth, J. A. Jones, and T. Freegarde, Composite pulses for interferometry in a thermal cold atom cloud, Phys. Rev. A 90, 033608 (2014).

[61] X. Rong, J. Geng, F. Shi, Y. Liu, K. Xu, W. Ma, F. Kong, Z. Jiang, Y. Wu, and J. Du, Experimental fault-tolerant universal quantum gates with solid-state spins under ambient conditions, Nat. Commun. 6, 8748 (2015).

[62] G. H. Low, T. J. Yoder, and I. L. Chuang, Methodology of Resonant Equiangular Composite Quantum Gates, Phys. Rev. X 6, 041067 (2016).

[63] S. Wu, T. Plisson, R. C. Brown, W. D. Phillips, and J. V. Porto, Multiphoton Magnetooptical Trap, Phys. Rev. Lett. 103, 173003 (2009).

[64] Y. Ma, X. Huang, X. Wang, L. Ji, Y. He, L. Qiu, J. Zhao, Y. Wang, and S. Wu, Precise pulse shaping for quantum control of strong optical transitions, Opt. Express 28, 17171 (2020).

[65] T. G. M. Freegarde, J. Walz, and T. W. Hänsch, Confinement and manipulation of atoms using short laser pulses, Opt. Commun. 117, 262 (1995).

[66] A. Goepfert, I. Bloch, D. Haubrich, F. Lison, R. Schütze, R. Wynands, and D. Meschede, Stimulated focusing and deflection of an atomic beam using picosecond laser pulses, Phys. Rev. A 56, R3354(R) (1997).

[67] M. J. Madsen, D. L. Moehring, P. Maunz, R. N. Kohn, Jr., L.-M. Duan, and C. Monroe, Ultrafast Coherent Excitation of a Trapped Ion Qubit for Fast Gates and Photon Frequency Qubits, Phys. Rev. Lett. 97, 040505 (2006).

[68] A. Monmayrant, S. Weber, and B. Chatel, A newcomer's guide to ultrashort pulse shaping and characterization, J. Phys. B 43, 103001 (2010).

[69] A. M. Weiner, Ultrafast optical pulse shaping: A tutorial review, Opt. Commun. 284, 3669 (2011).

[70] M. Garwood and L. DelaBarre, The return of the frequency sweep: Designing adiabatic pulses for contemporary NMR, J. Magn. Reson. 153, 155 (2001).

[71] G. Facchinetti, S. D. Jenkins, and J. Ruostekoski, Storing Light with Subradiant Correlations in Arrays of Atoms, Phys. Rev. Lett. 117, 243601 (2016).

[72] L. Corman, J. L. Ville, R. Saint-Jalm, M. Aidelsburger, T. Bienaimé, S. Nascimbène, J. Dalibard, and J. Beugnon, Transmission of near-resonant light through a dense slab of cold atoms, Phys. Rev. A 96, 053629 (2017).

[73] S. Jennewein, M. Besbes, N. J. Schilder, S. D. Jenkins, C. Sauvan, J. Ruostekoski, J.-J. Greffet, Y. R. P. Sortais, and A. Browaeys, Coherent Scattering of Near-Resonant Light by a Dense Microscopic Cold Atomic Cloud, Phys. Rev. Lett. 116, 233601 (2016). 
[74] P.-O. Guimond, A. Grankin, D. V. Vasilyev, B. Vermersch, and P. Zoller, Subradiant Bell States in Distant Atomic Arrays, Phys. Rev. Lett. 122, 093601 (2019).

[75] J. Javanainen and J. Ruostekoski, Light propagation beyond the mean-field theory of standard optics, Opt. Express 24, 993 (2016).

[76] I. Carusotto and C. Ciuti, Quantum fluids of light, Rev. Mod. Phys. 85, 299 (2013).

[77] D. E. Chang, V. Vuletić, and M. D. Lukin, Quantum nonlinear optics-photon by photon, Nat. Photonics 8, 685 (2014).

[78] C. Noh and D. G. Angelakis, Quantum simulations and manybody physics with light, Rep. Prog. Phys. 80, 016401 (2016).

[79] G. Reinaudi, T. Lahaye, Z. Wang, and D. Guéry-Odelin, Strong saturation absorption imaging of dense clouds of ultracold atoms, Opt. Lett. 32, 3143 (2007).

[80] K. Hueck, N. Luick, L. Sobirey, T. Lompe, H. Moritz, L. Clark, and C. Chin, Calibrating high intensity absorption imaging of ultracold atoms, Opt. Express 25, 8670 (2017).
[81] M. Gross and S. Haroche, Superradiance: An essay on the theory of collective spontaneous emission, Phys. Rep. 93, 301 (1982).

[82] J. T. Manassah, Cooperative radiation from atoms in different geometries: Decay rate and frequency shift, Adv. Opt. Photon. 4, 108 (2012).

[83] W. Guerin, M. O. Araújo, and R. Kaiser, Subradiance in a Large Cloud of Cold Atoms, Phys. Rev. Lett. 116, 083601 (2016).

[84] T. Bienaimé, N. Piovella, and R. Kaiser, Controlled Dicke Subradiance From a Large Cloud of Two-Level Systems, Phys. Rev. Lett. 108, 123602 (2012).

[85] R. T. Sutherland and F. Robicheaux, Coherent forward broadening in cold atom clouds, Phys. Rev. A 93, 023407 (2016).

[86] G. T. Genov, D. Schraft, T. Halfmann, and N. V. Vitanov, Correction of Arbitrary Field Errors in Population Inversion of Quantum Systems by Universal Composite Pulses, Phys. Rev. Lett. 113, 043001 (2014). 